\title{
High-order aberration compensation with multi-frame blind deconvolution and phase diversity image restoration techniques
}

\author{
G. B. Scharmer ${ }^{1,2}$, M. G. Löfdahl ${ }^{1,2}$, T. I. M. van Werkhoven ${ }^{1,3}$, and J. de la Cruz Rodriguez ${ }^{1,2}$ \\ 1 Institute for Solar Physics, Royal Swedish Academy of Sciences, AlbaNova University Center, 10691 Stockholm, Sweden \\ e-mail: scharmer@astro.su.se \\ 2 Stockholm Observatory, Dept. of Astronomy, Stockholm University, AlbaNova University Center, 10691 Stockholm, Sweden \\ 3 Sterrekundig Instituut Utrecht, Utrecht University, PO Box 80000, 3508TA Utrecht, The Netherlands
}

Received 15 April 2010 / Accepted 9 June 2010

\begin{abstract}
Context. For accurately measuring intensities and determining magnetic field strengths of small-scale solar (magnetic) structure, knowledge of and compensation for the point spread function is crucial. For images recorded with the Swedish 1-meter Solar Telescope (SST), restoration with multi-frame blind deconvolution (MFBD) and joint phase diverse speckle (JPDS) methods lead to remarkable improvements in image quality but granulation contrasts that are too low, indicating additional stray light.

Aims. We propose a method to compensate for stray light from high-order atmospheric aberrations not included in MFBD and JPDS processing.

Methods. To compensate for uncorrected aberrations, a reformulation of the image restoration process is proposed that allows the average effect of hundreds of high-order modes to be compensated for by relying on Kolmogorov statistics for these modes. The applicability of the method requires simultaneous measurements of Fried's parameter $r_{0}$. The method is tested with simulations as well as real data and extended to include compensation for conventional stray light.

Results. We find that only part of the reduction of granulation contrast in SST images is due to uncompensated high-order aberrations. The remainder is still unaccounted for and attributed to stray light from the atmosphere, the telescope with its re-imaging system and to various high-altitude seeing effects.

Conclusions. We conclude that statistical compensation of high-order modes is a viable method to reduce the loss of contrast occurring when a limited number of aberrations is explicitly compensated for with MFBD and JPDS processing. We show that good such compensation is possible with only 10 recorded frames. The main limitation of the method is that already MFBD and JPDS processing introduces high-order compensation that, if not taken into account, can lead to over-compensation.
\end{abstract}

Key words. techniques: high angular resolution - techniques: image processing - instrumentation: adaptive optics methods: numerical - atmospheric effects - Sun: granulation

\section{Introduction}

Since the emergence of 3D simulations of solar convection, there has remained a disturbing discrepancy between the measured contrast of granulation and that obtained from simulations. It has been suspected that the major reason for this discrepancy is atmospheric and telescope stray light. However, difficulties of accurately characterizing such stray light, in particular as regards the far wings of the corresponding point spread functions, have prevented firm conclusions. Questions about the accuracy of the predicted intensity contrasts obtained from simulations have also been raised and possible effects of magnetic fields proposed as explanation of the reduced contrast (Uitenbroek et al. 2007). Detailed comparisons of the shapes of observed and simulated spectral lines, determined by correlations between Doppler velocities and temperature variations, however, show a remarkable agreement (Nordlund et al. 2009). In addition, there is now excellent agreement between granulation rms contrasts obtained with independently developed 3D MHD codes (Wedemeyer-Böhm \& Rouppe van der Voort 2009).

Recent data obtained from Hinode show higher granulation contrasts than obtained from most ground based solar telescopes, in spite of the relatively modest aperture diameter of the solar optical telescope (SOT) on Hinode (Wedemeyer-Böhm \& Rouppe van der Voort 2009). The combined PSF of the Broadband Filter Imager (BFI) and SOT, based on images recorded during a Mercury transit and a solar eclipse and including stray light and the effects of the large central obscuration and spider, was determined by Wedemeyer-Böhm (2008). The obtained PSF leads to reconstructed granulation contrasts that are remarkably close to those of 3D simulations (Wedemeyer-Böhm \& Rouppe van der Voort 2009). A simpler fit of the PSF in terms of 4 Gaussians also leads to good agreements between granulation contrast measured with BFI on Hinode and MHD simulations (Mathew et al. 2009). In addition, agreement between simulated images degraded to the resolution of the SOT spectropolarimeter (SP) shows good agreement, even without stray light correction (Danilovic et al. 2008). This finally should settle any remaining controversies about the rms granulation contrasts obtained from 3D solar convection simulations.

In this paper, we initiate a search for the origin of the "missing" granulation contrast in images observed with the Swedish 1-m Solar Telescope (SST) and restored with methods based on multi-frame blind deconvolution (MFBD; Löfdahl 2002), such as joint phase diverse speckle (JPDS; Paxman et al. 1992) and multi-object MFBD (MOMFBD; van Noort et al. 2005). 
We have previously observed the effects of truncating the wavefront expansion on contrasts and power spectra when comparing data restored with MFBD based methods to data restored with speckle interferometry (Paxman et al. 1996; Rouppe van der Voort et al. 2004), and this observation is the basis of the proposed investigation.

An important motivation for this study is Stokes measurements: Magnetic structures inside and outside sunspots have much more fine-structure than granulation. High spatial resolution and low stray light is required for accurate field strength measurements of such structures. Present inversion techniques, such as Helix (Lagg et al. 2004), allow pixel-to-pixel compensation for stray light through a parameter determined by the fits to the observed Stokes profiles. It is however obvious that a stray light PSF cannot vary significantly from one pixel to another and that the preferred approach is compensation for stray light in preprocessing and to carry out the inversions without allowance for stray light.

We note that polarization signals from isolated small-scale structures recorded with high spatial resolution and high signalto-noise $(\mathrm{S} / \mathrm{N})$ show a halo of polarized light, probably originating from uncorrected aberrations and/or stray light. Such isolated magnetic structures provide useful "point sources" that can be used to validate measured PSFs in addition to measurements of granulation contrast at different wavelengths.

The paper is organized as follows: We describe the MFBD/JPDS imaging model and reconstruction in Sect. 2 and propose a method for compensation of the PSF for high-order modes in Sect. 3. We describe simulations and tests made to validate the proposed method in Sect. 4 and apply the method to SST data in Sect. 5. Finally, we extend the method to include conventional stray light in Sect. 6 and summarize the results in Sect. 7.

\section{MFBD imaging model and image reconstruction}

To allow compensation for aberrations not accounted for in processing with MFBD based methods, we need to briefly review the imaging model and image reconstruction process used. Although the MFBD based methods include sophisticated schemes involving objects observed at different wavelengths (MOMFBD) and with known aberration differences (JPDS), the basic imaging model is simple and the reconstruction of the image well defined.

In the MOMFBD implementation of van Noort et al. (2005) the imaging process is modeled as a space-invariant (valid for sufficiently small sub-fields) convolution between an unknown object $f$ and a point spread function $t_{k}$, the Fourier transforms of which are $F$ and $T_{k}$, where the index $k$ corresponds to a particular exposure. An additive Gaussian noise term $n_{k}$ is assumed ${ }^{1}$. The Fourier transform $D_{k}$ of the observed image $d_{k}$ is then related to $F, T_{k}$ and $N_{k}$ via

$D_{k}=F T_{k}+N_{k}$.

The assumption of additive Gaussian noise leads to a maximumlikelihood estimate of the object and transfer functions, equivalent to the solution of a conventional non-linear least-squares

\footnotetext{
1 A maximum-likelihood estimate with a Poisson noise model (Paxman et al. 1992) would be more appropriate when photon noise is the dominating source of modelling error. The optimization process for the Poisson noise model is computationally much more demanding than for the additive Gaussian noise model.
}

fit problem, and corresponds to the minimization of the scalar quantity $L$,

$L=\sum_{u, v} \sum_{k=1}^{K}\left|D_{k}-\hat{F} \hat{T}_{k}\right|^{2}$,

where $u, v$ represents pixels in Fourier space. This equation directly leads to an optimum estimate $\hat{F}$ of the true object $F$, expressed in terms of the $K$ estimated transfer functions $\hat{T}_{k}$ and associated observed images $d_{k}$

$\hat{F}=\left.\sum_{k=1}^{K} D_{k} \hat{T}_{k}^{*}\left|\sum_{k=1}^{K}\right| \hat{T}_{k}\right|^{2}$,

(Paxman et al. 1992), where $\left|\hat{T}_{k}\right|^{2}=\hat{T}_{k} \hat{T}_{k}^{*}$ and $\hat{T}_{k}^{*}$ is the complex conjugate of $\hat{T}_{k}$. Note that Eq. (3) can be written as

$\hat{F}=\sum_{k=1}^{K} w_{k} D_{k} / \hat{T}_{k}$

where $D_{k} / \hat{T}_{k}$ are estimates of the object $F$ based on single frames and $w_{k}$ are weights, given by

$w_{k}=\left.\left|\hat{T}_{k}\right|^{2}\left|\sum_{k=1}^{K}\right| \hat{T}_{k}\right|^{2}$.

and $\sum w_{k}=1$. This emphasizes that multi-frame deconvolution assigns weights given by the absolute squares of the estimated transfer functions associated with each image and at each spatial frequency. This is the optimum way of combining measurements for which $S / N$ varies because the transfer functions vary from one frame to another while the rms noise is the same for all measurements. In particular, zero weight is given to spatial frequencies for which the estimated transfer function pass through zero for an individual frame, thus avoiding division by zero as may happen when $D_{k}$ is directly divided by $\hat{T}_{k}$ to obtain a restored object. Equation (4) also means that a good image $D_{k}$, corresponding to an overall large $\left|\hat{T}_{k}\right|$, is given (much) higher weight than a poor image in the reconstruction of the object $F$. This weighting introduces a bias in the image reconstruction that is of importance when compensating the images for uncorrected high-order aberrations.

\section{Compensation for uncorrected high-order aberrations}

MFBD and related image reconstruction techniques do not rely on statistical properties of seeing, thereby allowing compensation for individual telescope aberrations and the effects of an adaptive optics (AO) system in addition to seeing-induced aberrations. By estimating the transfer function for each recorded frame, a small number of exposures can be used to restore the object. However, the number of aberration coefficients that can be determined from focused and defocused images is necessarily limited. In less than perfect seeing, this leaves a partially compensated PSF with enhanced wings. This corresponds to the well-known "halo" seen in images of point-like objects recorded with night-time telescopes and partial AO compensation of seeing (e.g., Conan et al. 1992). 


\subsection{Seeing statistics and residual aberrations expected}

To appreciate the importance of residual high-order aberrations, we note that Danilovic et al. (2008) found that aberrations as small as 0.044 waves, corresponding to nearly perfect optics with a Strehl ratio of $93 \%$, reduces the measured rms contrast of granulation from $8.4 \%$ to $8.1 \%$ with Hinode's spectropolarimeter. Thus, within the framework of the present investigation, we clearly should consider any residual aberrations that decrease the Strehl ratio below $90 \%$.

The effect of partially corrected aberrations on the Strehl ratio is well-known from the work of Fried, Noll, Wang, Markey and others. We refer to Roddier (1999) for an overview of relevant results. The residual wavefront variance for zonal correction can be estimated as

$\sigma^{2}=0.34\left(D / r_{0}\right)^{5 / 3} N^{-5 / 6}$,

where $\sigma$ is in radians, $D$ is the telescope diameter, $r_{0}$ is Fried's parameter, and $N$ is the number of independently corrected aberration parameters. Optimum compensation with a given number of degrees of freedom is obtained with Karhunen-Loève (KL) modes ${ }^{2}$. The residual wavefront variance, estimated from a fit to data plotted in Fig. 3.1 of Roddier (1999), is approximately

$\sigma^{2}=0.3\left(D / r_{0}\right)^{5 / 3} N^{-0.92}$.

The Strehl ratio $R$ can be estimated as

$R=\exp \left(-\sigma^{2}\right)$.

We shall in the following estimates assume perfect zonal correction, which in the case of AO correction would represent an unrealistically high efficiency. For the SST, with a telescope diameter of $0.98 \mathrm{~m}$, we find that $R=0.81$ with $N=36$ aberration parameters when $r_{0}=22 \mathrm{~cm}$ and that $R>0.9$ only when $r_{0}>33 \mathrm{~cm}$. With good seeing corresponding to $r_{0}=10 \mathrm{~cm}\left(1^{\prime \prime}\right.$ seeing) and very good seeing to $r_{0}=14 \mathrm{~cm}(0.7$ seeing), we need to compensate about 400 respectively 200 modes to reach a Strehl ratio of about 0.9 . When $r_{0}=20 \mathrm{~cm}(0 . ' 5$ seeing), we need to correct nearly 100 modes. Thus even with excellent seeing, we should be concerned about the effects of uncorrected aberrations in MFBD processing. These estimates also imply that the problem of compensation of high-order aberrations in solar imaging is primarily related to near-ground seeing, associated with a large isoplanatic patch, since $r_{0}$ is typically more than a factor 3 smaller for the ground layer than for the high-altitude layers during day-time.

An important limitation at short wavelengths is that $r_{0}$ scales as $\lambda^{6 / 5}$. When $r_{0}=20 \mathrm{~cm}$ at $\lambda=630 \mathrm{~nm}, r_{0}$ is only $11 \mathrm{~cm}$ at $390 \mathrm{~nm}$. In this case we need to compensate nearly 100 aberrations at $630 \mathrm{~nm}$ and over 300 aberrations at $390 \mathrm{~nm}$ to reach a Strehl ratio of 0.9. Even in excellent seeing, images recorded and processed with conventional MFBD at $390 \mathrm{~nm}$ will be far from perfectly corrected. It is therefore not surprising that measured rms contrasts of granulation with the SST show much larger discrepancies with 3D simulations at short wavelengths than at long wavelengths.

\subsection{Compensation for high-order aberrations}

In MOMFBD processing of SST data, particularly from the CRISP instrument (Scharmer et al. 2008), a data set corresponding to a line scan can consist of $\sim 1000$ exposures in several cameras. However, an estimated object from a particular wavelength

\footnotetext{
2 This is however not true for MFBD/JPDS restoration of images obtained with AO.
}

and polarization state is typically based on deconvolution of a relatively small number of images $(\sim 10)$, degraded by residual low-order aberrations from partial correction with a 37-electrode AO system and uncorrected high-order atmospheric aberrations. As long as MFBD processing compensates the residual aberrations partially corrected by the AO system, we are to some extent justified in ignoring the corrections made by the AO system: It is the residual aberrations after that AO correction that define the $\mathrm{S} / \mathrm{N}$ of individual images and the weighting implied by Eq. (4).

Our goal is to estimate the effect on the transfer function of uncompensated high-order aberrations, defined in Eq. (11). To do this, we will assume that these modes have amplitudes given by the assumption of Kolmogorov turbulence. This is the same basic assumption as used in Speckle Interferometry, but with the difference that low-order modes are compensated individually and for each exposed frame with MFBD. We conjecture that:

- there will be rather small variations of the PSF from one frame to another because residual wavefront errors, after correction on the order of 30 modes, depend on the accumulative effect of hundreds of modes rather than a few largeamplitude low-order modes;

- a relatively small number of frames is therefore needed to obtain stable averages.

Solar speckle techniques rely on an average theoretical transfer function obtained from Kolmogorov turbulence statistics and based on a large number of recorded frames. This corresponds to compensation, in an average sense, for an infinite number of aberration coefficients. Recent developments allow solar speckle processing also of AO corrected images (Puschmann \& Sailer 2006; Wöger 2007) but (as far as we know) present solar speckle techniques do not include compensation for telescope aberrations.

\subsubsection{The proposed method}

Our goal is to develop a method that combines the advantages of MFBD/JPDS and speckle methods. The approach taken is to compensate individual frames for low-order modes and to add a compensation for the average effect of hundreds of high-order aberrations from atmospheric turbulent seeing.

This compensation can be implemented in various ways. We have chosen the following simple approach that has the advantage of not involving the observed images but only their corresponding transfer functions. We note that information about the "true" (exact) transfer functions and the true object are encoded in the observed images. Ignoring the noise term in Eq. (1) and combining with Eq. (3), we obtain a relation between the estimated object $\hat{F}$, the true object $F$, and the corresponding exact and estimated transfer functions $T_{k}$ and $\hat{T}_{k}$

$\hat{F}=\left.F \sum_{k=1}^{K} T_{k} \hat{T}_{k}^{*}\left|\sum_{k=1}^{K}\right| \hat{T}_{k}\right|^{2}$.

We note that this is a relation of the form

$\hat{F}=S F$

i.e., in the form of a multiplication of the true object with a transfer function,

$S=\left.\sum_{k=1}^{K} T_{k} \hat{T}_{k}^{*}\left|\sum_{k=1}^{K}\right| \hat{T}_{k}\right|^{2}=\sum_{k=1}^{K} w_{k} \frac{T_{k}}{\hat{T}_{k}}$, 
that is a weighted average of the ratio of the true and estimated transfer equations. This equation does not solve the problem unless the exact transfer functions are known. However, we can estimate $S$ by including known statistical properties of atmospheric high-order aberrations while using MFBD or JPDS estimates of low-order aberrations for each individual exposure. We propose the following estimate of $S$ from a combination of aberration parameters determined by MFBD/JPDS processing and statistical properties of atmospheric seeing:

$$
\hat{S}=\sum_{k=1}^{K}\left\langle\left. T_{k} \hat{T}_{k}^{*}\left|\sum_{n=1}^{K}\right| \hat{T}_{n}\right|^{2}\right\rangle=\left.\sum_{k=1}^{K}\left\langle T_{k}\right\rangle \hat{T}_{k}^{*}\left|\sum_{k=1}^{K}\right| \hat{T}_{k}\right|^{2}
$$

where angular brackets, $\langle\ldots\rangle$, indicate an ensemble average over many independent realizations for each $k$ separately and where $T_{k}$ contains the same low-order aberrations, obtained with MFBD or JPDS processing, as $\hat{T}_{k}$.

To estimate the effect of high-order aberrations in $T_{k}$, we add random higher-order KL modes with amplitudes given by Kolmogorov statistics and average the transfer equation over many realizations to obtain stable averages. The proposed method requires measurements of $r_{0}$ at the time of recording the data. The preferable method for accurate measurements of $r_{0}$ is via data from an open-loop wavefront sensor located before the adaptive mirror, as implemented at the SST (Scharmer $\&$ van Werkhoven 2010). It is also possible, as done at the Dunn telescope diameter (Marino et al. 2004), to combine closed-loop wavefront sensor data with the control matrix and output voltages to estimate $r_{0}$ and residual low-order aberrations. The quality of such measurements are to some extent limited by time delays and inaccuracies in the control matrix, but experience with night-time AO systems clearly indicates that good PSF compensation is indeed possible with such data (Veran et al. 1997).

\section{Simulations}

To investigate the feasibility of the proposed method for compensation of missing high-order aberrations in MFBD and JPDS processing, numerical calculations and simulations were made.

\subsection{Ideal compensation with point sources}

We used KL functions based directly on the theory of Fried (1978), as implemented by Dai (1995). These functions are orthogonal on a circular aperture and statistically independent for Kolmogorov turbulence. For such turbulence, the variances depend only on $r_{0}$. To produce random wavefronts following Kolmogorov statistics, 1001 random numbers were drawn from a standard normal distribution, scaled with the square root of the theoretical variances and used as coefficients for KL functions 4-1004 (in decreasing variance order). The resulting wavefronts were scaled to different values of Fried's parameter $r_{0}$. To simulate the effect of partial correction with an efficient AO system, we reduced the amplitudes of KL coefficients 4-37 with a factor 4 (this represents an overestimate of the actual efficiency). Piston (coefficient 1) is ignored because it does not contribute to the OTFs and tip-tilt correction (coefficients 2 and 3) was assumed to be perfect, corresponding to images recorded with sufficiently short exposure times to remove changes in image position and blurring during the exposure.

The residuals of the AO-corrected aberrations were used to represent the estimate of the transfer equations $\hat{T}_{k}$. For this calculation we assumed 10 observed images with independently obtained wavefronts. We computed the "corrective" transfer function $S$ from Eq. (11) by using the actual high-order aberrations corresponding to each $T_{k}$ and then the approximate version, $\hat{S}$, using the statistical averages (based on 100 realizations of the high-order tail) of the transfer functions, as defined in Eq. (12). We finally compared the exact and estimated transfer functions $S$ and $\hat{S}$. This corresponds to perfect MFBD correction of the first $36 \mathrm{KL}$ modes and perfect knowledge of $r_{0}$.

Figure 1 shows the PSFs corresponding to $S$ and $\hat{S}$, scaled such that the wings of the PSFs can be seen, for values of $r_{0}$ in the range $5-25 \mathrm{~cm}$ and $\lambda=630 \mathrm{~nm}$. Calculations were made for a $98 \mathrm{~cm}$ telescope diameter, corresponding to the SST, with critical sampling at $\lambda=630 \mathrm{~nm}$, corresponding to 0. '.066/pixel. The field of view (FOV) shown is 4 ". $2 \times 4$ ". 2 . As expected, the true PSFs show a speckled structure in the wings, but smoothed by the averaging effect obtained by combining ten images. The corresponding approximate PSFs show much less structure in the wings but are otherwise similar to the actual PSFs. It can also be seen that the effective diameter of the PSF, defined as that containing $90 \%$ of the energy of the PSF, increases with decreasing $r_{0}$. When $r_{0}$ equals $25 \mathrm{~cm}$, that diameter is approximately $1^{\prime \prime}$. 1 , when $r_{0}$ is $5 \mathrm{~cm}$, it increases to approximately $4^{\prime \prime 3}$. Figure 2 shows the encircled energy as function of radius for the approximate and exact PSFs. It is clear that the encircled energy of the approximate PSF follows that of the actual PSF nearly exactly. In Fig. 3 we show calculated Strehl ratios of the PSFs as function of $r_{0}$. The exact and approximate PSFs give nearly exactly the same Strehl ratios and also agree well with what is expected for perfect KL correction of 36 modes from Eqs. (7) and (8). Finally, Fig. 4 shows radially averaged power spectra for the transfer functions corresponding to the exact and approximate PSFs, again showing an excellent agreement between the two at all spatial frequencies. We also refer the reader to Fig. 12 by Rouppe van der Voort et al. (2004), where similar effects of seeing on observed power spectra of penumbral fine structure are discussed.

\subsection{Ideal compensation with granulation images}

To investigate the effects of uncompensated high-order aberrations on granulation images, we used synthetic images calculated from a field-free 3D MHD simulation (Stein \& Nordlund 1998); these simulation data were kindly provided by Mats Carlsson. The synthetic images were calculated at a wavelength of $630 \mathrm{~nm}$ and were degraded to a resolution corresponding to $90 \%$ of the diffraction limit of the SST while keeping the image scale of the original synthetic images. The images were then degraded by Fourier multiplication with $S$ based on 10 wavefronts. This corresponds to an MFBD estimate $\hat{F}$ based on 10 observed images. $\hat{F}$ was then corrected using an approximate $\hat{S}$ based on the same low-order aberrations as $S$ and a high-order compensation with $1000 \mathrm{KL}-$ modes according to Eq. (12). The original, degraded and restored images are shown in Fig. 5 together with their rms contrasts. Note that the degraded images differ from the original image in contrast but not in fine structure shown. The main effect of the higher-order aberrations is to add stray light, decreasing the rms contrast, whereas all small-scale features of the original image are retained and restored to full

\footnotetext{
3 As discussed in Sect. 5, the increasingly larger diameter of the PSF in degrading seeing restricts the use of very small subfields needed with MFBD/JPDS methods to deal with anisoplanatism. A similar restriction applies when the images are intentionally degraded by including a defocused imaging channel, used with JPDS processing.
} 
G. B. Scharmer et al.: High-order aberration compensation with MFBD and PD image restoration techniques

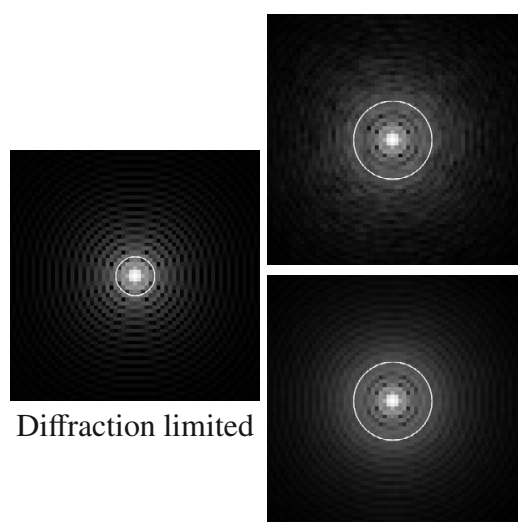

$r_{0}=25 \mathrm{~cm}$

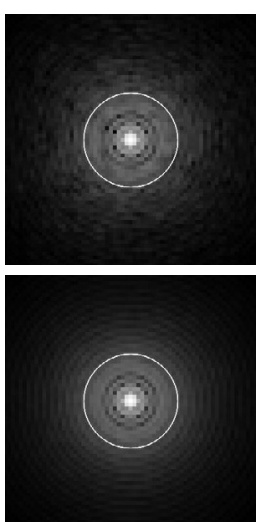

$20 \mathrm{~cm}$
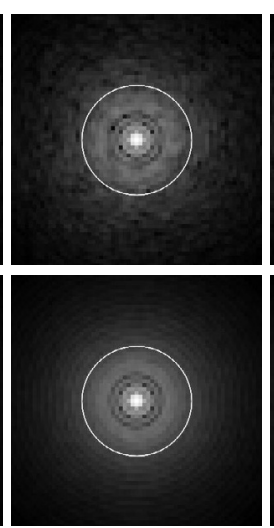

$15 \mathrm{~cm}$
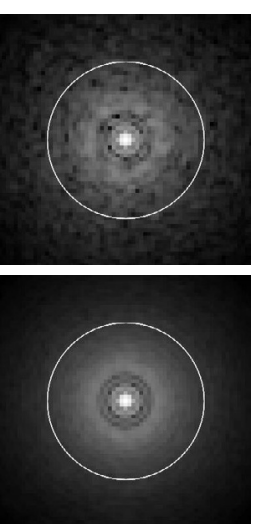

$10 \mathrm{~cm}$

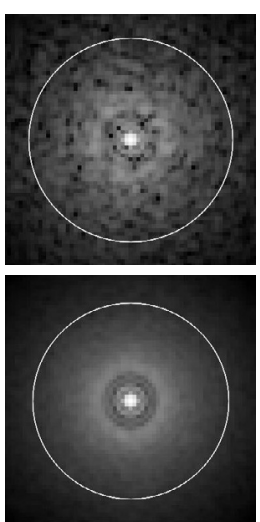

$7 \mathrm{~cm}$

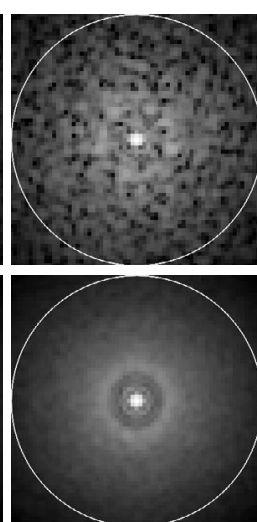

$5 \mathrm{~cm}$

Fig. 1. PSFs displayed in log scale. The circles mark $90 \%$ encircled energy, see also Fig. 2. Far left: Diffraction limited. Top: PSFs corresponding to $S$, i.e., true residual high-order aberrations for different $r_{0}$ as indicated. Bottom: Approximate PSFs, corresponding to $\hat{S}$, i.e., to the method proposed. The FOV shown is 4". $2 \times 4$ "' $2(64 \times 64$ pixels). The synthetic PSFs shown were calculated from 10 individual noise-free frames and with perfect compensation for the 36 lowest KL-modes.

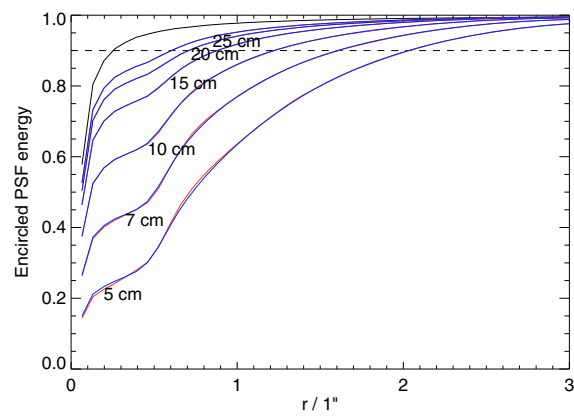

Fig. 2. Encircled PSF energy for different $r_{0}$ as indicated in the figure. Red: PSFs based on $S$; Blue: PSFs based on $\hat{S}$; Black: diffraction limited PSF; Black dashed: 90\% level.

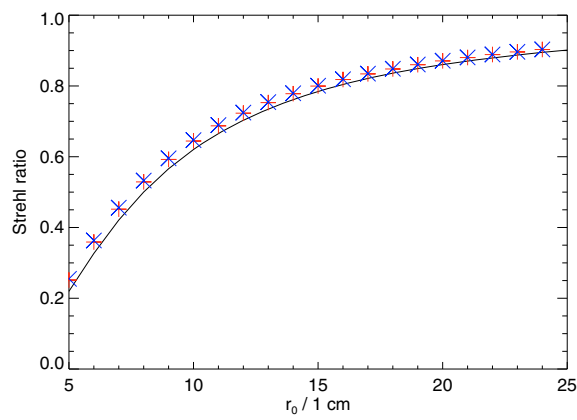

Fig. 3. Strehl ratios as a function of $r_{0}$. Solid line: Eqs. (7) and (8) with $N=37$; Red plus $(+)$ symbols: PSFs based on $S$; Blue cross $(\times)$ symbols: PSFs based on $\hat{S}$.

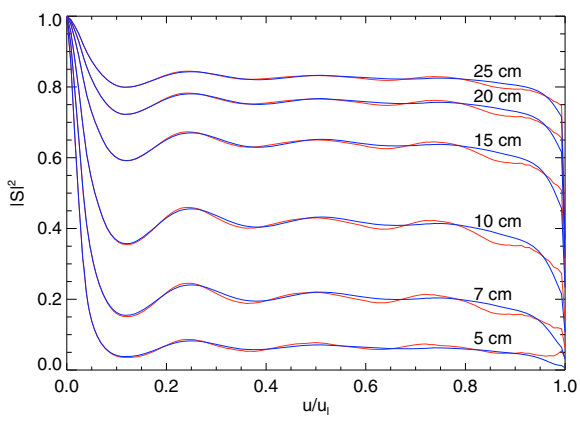

Fig. 4. Power spectra (angular averages) of $S$ (red) and $\hat{S}$ (blue) for $r_{0}$ as indicated.

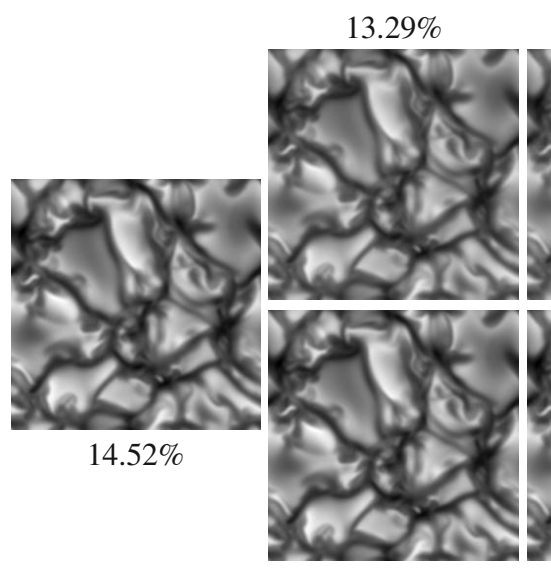

$14.51 \%$
$12.78 \%$

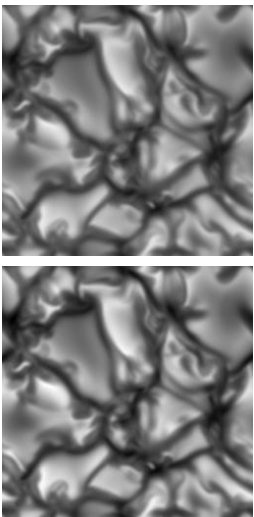

$14.51 \%$
$11.83 \%$

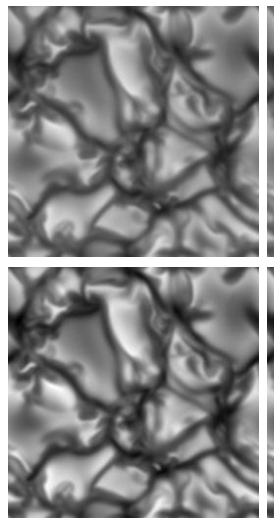

$14.50 \%$
$9.77 \%$

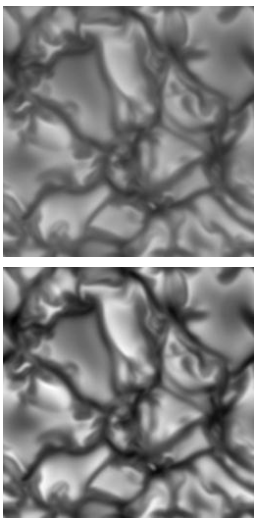

$14.48 \%$
$7.23 \%$

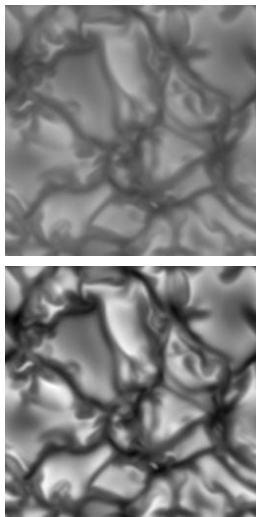

$14.41 \%$
$4.63 \%$

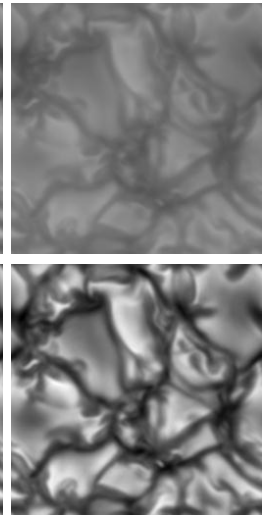

$14.29 \%$

Fig. 5. Synthetic images calculated at a wavelength of $630 \mathrm{~nm}$. Far left: Original image. Top: Low-pass images degraded by high-order aberrations, i.e., by $S$, corresponding to $r_{0}=25,20,15,10,7$ and $5 \mathrm{~cm}$, resp. (same layout as Fig. 1). Bottom: Degraded images compensated by use of the method described, i.e., by $\hat{S}$. All images are scaled between min and max of the original image and low-pass filtered to $90 \%$ of the SST diffraction limit. The numbers above and below the image tiles are the rms contrasts in percent of the mean intensity $(100 \times \mathrm{rms} / \mathrm{mean})$.

contrast with the approximate PSFs. As shown in Fig. 5, the loss in contrast is from $14.5 \%$ to $11.8 \%$ when $r_{0}$ is $15 \mathrm{~cm}$, or a reduction of the rms contrast by nearly $20 \%$. This corresponds to very good seeing conditions. In more typical seeing conditions, when $r_{0}$ is $10 \mathrm{~cm}$ or smaller, the effects are larger.
We conclude that whereas details of the exact and approximate PSFs certainly differ, the overall effect of the high-order aberrations is to add spatial stray light to the images. The proposed method for statistical compensation of high-order aberrations in principle should work very well. 


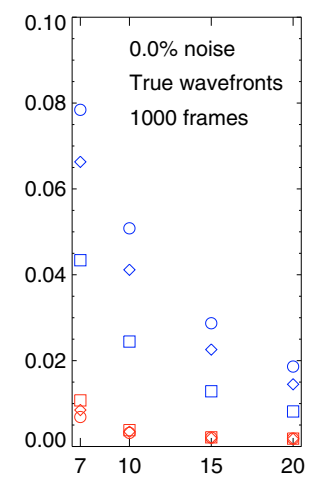

(a)

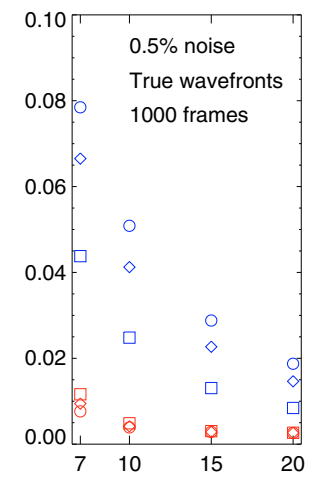

(g)

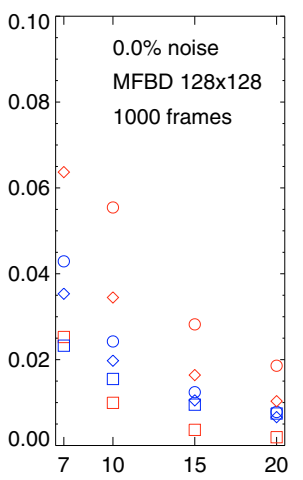

(b)

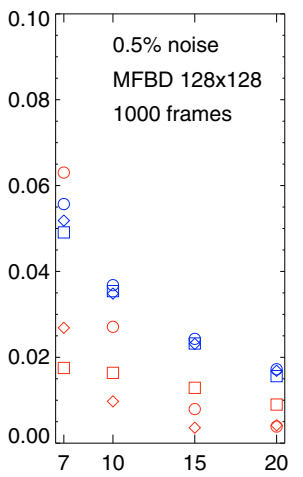

(h)

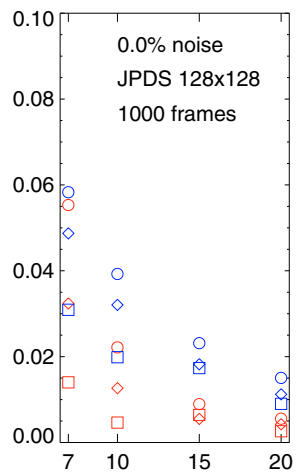

(c)

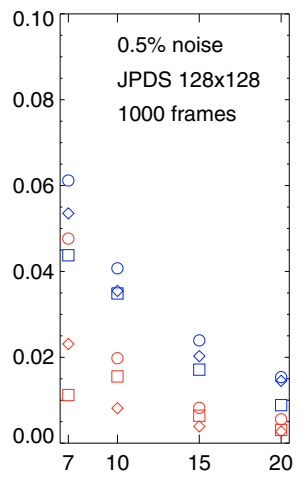

(i)

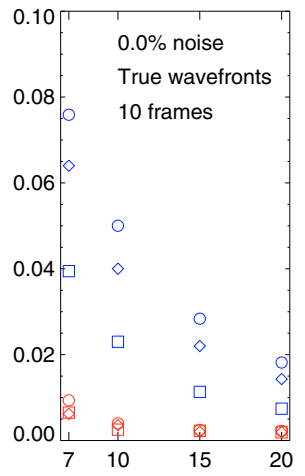

(d)

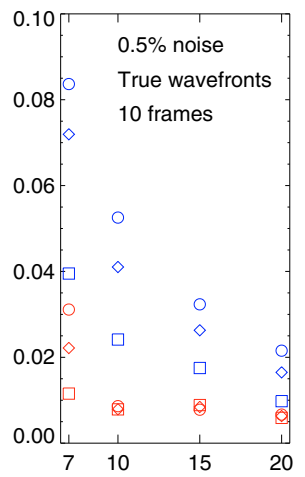

(j)

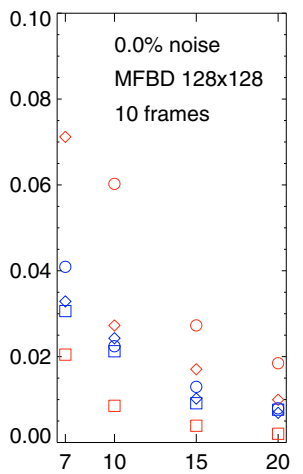

(e)

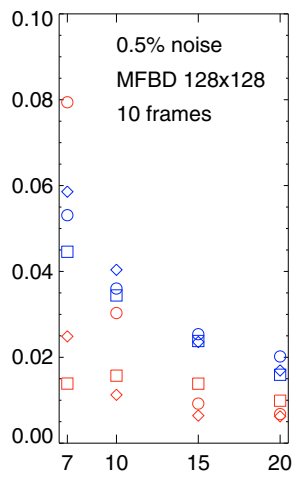

(k)

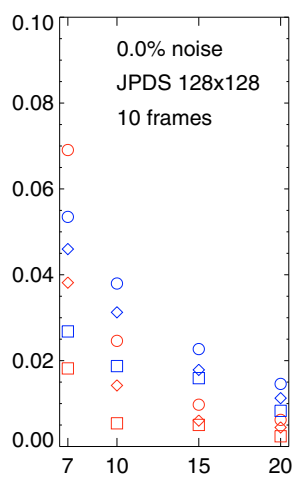

(f)

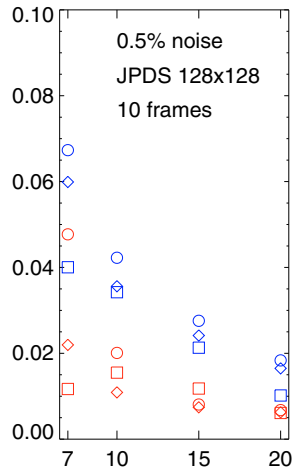

(1)

Fig. 6. RMS intensity error (in $\%$ of the average intensity) of restored granulation images as a function of $r_{0}$ (in $\mathrm{cm}$ ) after correcting $M$ modes. Circles (O): $M=35$; Diamonds $(\diamond): M=50$; Squares ( $\square)$ : $M=100$. Blue: Restored without $\hat{S}$ compensation; Red: Restored with $\hat{S}$ compensation.

\subsection{Inversion tests with granulation images}

With perfect determination of the low-order modes, correction of the high order modes in a statistical sense gives excellent results. However, we also need to investigate the effect of realistic errors from the low-order modes estimated with MFBD and JPDS techniques.

For this test, we calculated 1000 random wavefronts corresponding to Kolmogorov statistics, with reduced amplitudes for the first $36 \mathrm{KL}$ coefficients as described above. These wavefronts, scaled to different $r_{0}$, were used to construct sets of degraded granulation images with and without phase diversity, corresponding to an added focus shift, of 1 wave peak-to-valley. We used synthetic images calculated from the 3D MHD code as true objects, compressed by a factor 2 (by cropping the Fourier transform of the image). The image scale was set to match that of SST/CRISP during the 2009 observing season, 0.'059/pixel, corresponding to about $12 \%$ oversampling at $630 \mathrm{~nm}$.

The degraded synthetic images were processed with the MOMFBD program in various ways (MFBD or JPDS, different numbers of realizations, different subfield sizes (256 pixels $=15^{\prime \prime}, 128$ pixels $=77^{\prime \prime} 6,80$ pixels $\left.=4{ }^{\prime \prime} \cdot 7\right)$, different number of estimated wavefront parameters, with and without added noise).

\subsubsection{Results without $\hat{S}$ compensation}

Figure 6 shows rms intensity errors of restored images using different techniques (MFBD, JPDS), number of aberration parameters $(M=35,50$ and 100) with and without $\hat{S}$ compensation for different values of Fried's parameter $r_{0}$. We emphasize that for all calculations made, 1000 frames were used to obtain the wavefronts but in panels $\mathrm{d}-\mathrm{f}$ and $\mathrm{j}-\mathrm{l}$ subsets of only 10 frames were used to restore the images. This corresponds to processing of SST/CRISP data, where the broad-band channel of a MFBD or JPDS data set corresponds to on the order of 5001000 images. The simultaneously exposed narrow-band CRISP images are divided into typically 10-12 wavelength bins, each with 4 polarization bins. These narrow-band images are restored individually, but using the aberrations (mainly) determined by the 1000 broadband images. The upper row of plots show the results obtained without noise and the bottom row show the corresponding results with $0.5 \%$ Gaussian noise added to the simulated images.

Discussing first the results without $\hat{S}$ compensation (blue symbols), our reference for comparison is the results obtained using 1000 frames to restore the image and the first $M$ true (exact) aberration parameters (panel a). This shows the expected behavior: The quality of the restored images improves with increasing value of $r_{0}$ and also with increasing number of estimated aberration parameters used to restore the images. When $r_{0}$ is $20 \mathrm{~cm}$, the rms error with 50 perfectly known aberration parameters is only $1.5 \%$. However, in more typical seeing conditions ( $r_{0}=10 \mathrm{~cm}$ or smaller), the corresponding rms error is $2.5-8 \%$, depending on the number of aberration parameters compensated and the seeing quality.

Figure 6b (MFBD processing with 1000 frames) demonstrates that the rms intensity error obtained using only focused images to estimate the aberration parameters shows quite small variation of the intensity error with the number of aberration parameters $M$, when $M$ is in the range 35-100. The rms intensity error for $M=35$ MFBD calculations actually corresponds to what was obtained with the true aberration parameters for 


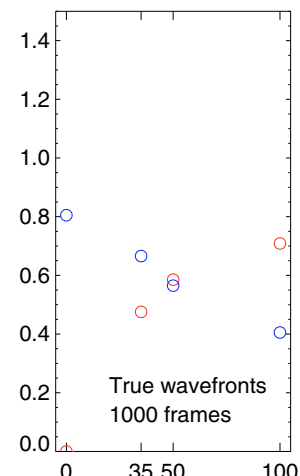

(a)

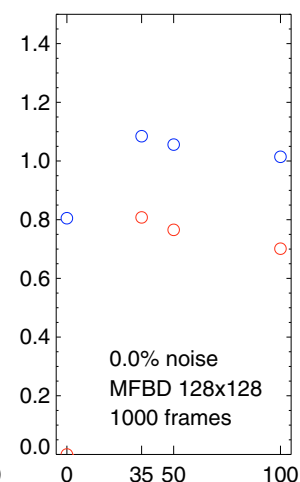

(b)

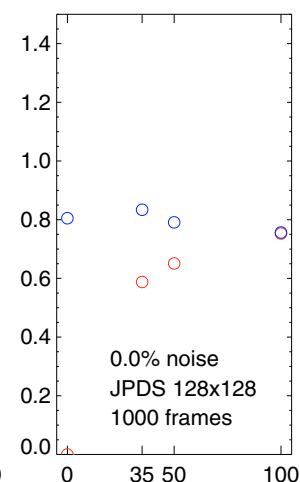

(c)

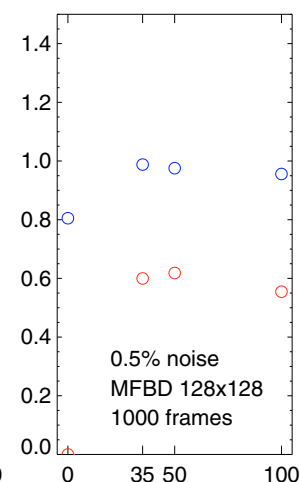

(d)

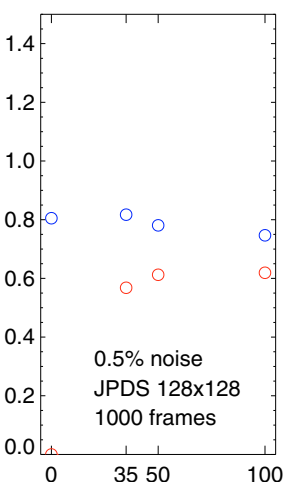

(e)

Fig. 7. Wavefront quantities as a function of $M$ (number of corrected modes) for $r_{0}=10 \mathrm{~cm}$. Red: rms wavefront in rad; Blue: rms wavefront residual in rad.

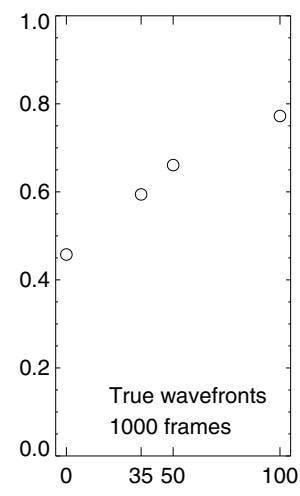

(a)

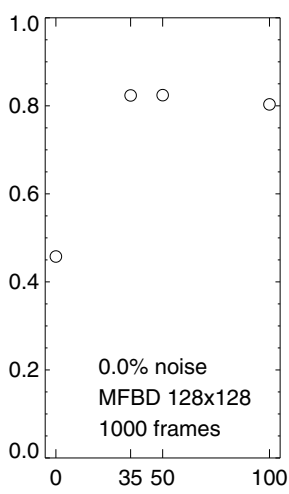

(b)

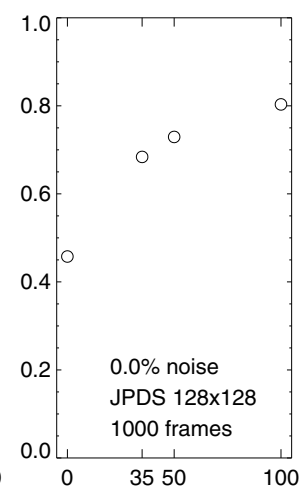

(c)

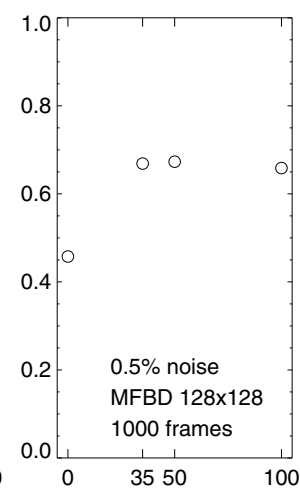

(d)

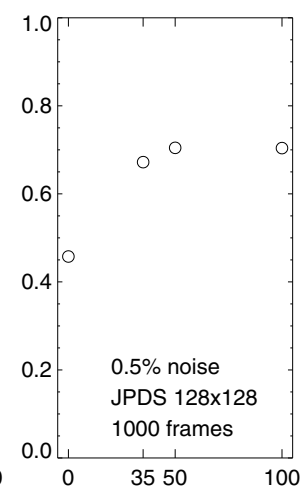

(e)

Fig. 8. Strehl ratio as a function of $M$ (number of corrected modes) for $r_{0}=10 \mathrm{~cm}$.

$M=100$ (Panel a)! This implies that MFBD processing with a given number of aberration parameters leads to estimates of the transfer function that compensate for the effects of missing higher-order aberration parameters (truncation of the wavefront). Increasing the number of estimated aberration parameters from 35 to 50 or 100 , reduces the efficiency of this compensation such that the reduction of the intensity error is relatively modest. Quite clearly, the estimated wavefront with MFBD processing must be inaccurate, but such that the estimated transfer function accurately represents the true transfer function. This conclusion is further supported by Fig. 7, which shows the variation of the (estimated) wavefront rms and wavefront error for different number of estimated wavefront parameters when $r_{0}=10 \mathrm{~cm}$. Panel a (red symbols) shows that the rms of the true wavefront increases by $50 \%$ when $M$ is increased from 35 to 100 . In contrast, the rms of the estimated wavefront for the $M$ first aberration parameters is strongly overestimated for $M=35$ and $M=50$ with MFBD processing such that the estimated wavefront rms is nearly independent of $M$. Thus, using a low-order or high-order representation for the wavefront leads to nearly the same estimated wavefront rms. A striking result is that MFBD processing of the first 35-100 corrected modes leads to residual wavefront errors (blue symbols) that are larger than if the wavefront is estimated to be exactly zero!

When using also a defocused channel (JPDS processing), the wavefronts are more constrained to represent reality, leading to smaller wavefront errors (Fig. 7c). But this constraint also limits the freedom in compensating the transfer function for high-order aberrations, such that larger intensity errors are obtained in the restored images with JPDS (Fig. 6c) than with MFBD processing (Fig. 6b).

These conclusions about differences between MFBD and JPDS processing are supported by the Strehl ratios calculated from wavefronts determined with the granulation images and shown in Fig. 8. With true aberration parameters (Fig. 8a) and without $\hat{S}$ compensation, the Strehl intensity increases gradually with the number of compensated aberration parameters $M$. However, with MFBD processing, the Strehl ratio is constant when $M$ is in the range $35-100$. The Strehl ratio achieved, $R=0.8$, is close to what is expected from Eqs. (6) and (8) for $N=M=100$. The variation of the Strehl ratio with $M$ obtained with JPDS processing is intermediate to that of MFBD and using the true aberration parameters.

Adding $0.5 \%$ Gaussian noise to the MFBD data shows that the results are sensitive to noise (Figs. 6h-i) and indicates that in practice the compensation effects discussed above will not be large and that the quality of MFBD and JPDS image restorations should be rather similar. The rms intensity error improvement is limited to that corresponding to about 50 "true" aberration parameters, or a Strehl ratio of 0.7 . Using more than 50 aberration parameters to represent the wavefront does not significantly improve the quality of the restored images.

\subsubsection{Results with $\hat{S}$ compensation}

The results obtained with $\hat{S}$ compensation are shown with red symbols in Fig. 6. With such compensation, restorations with "true" low-order wavefronts allow nearly perfectly restored 


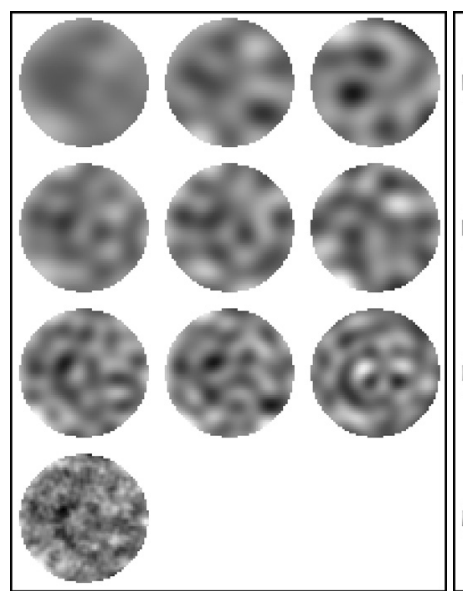

(a)

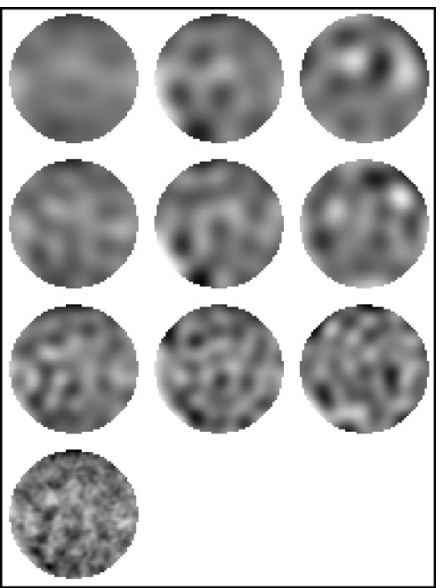

(b)

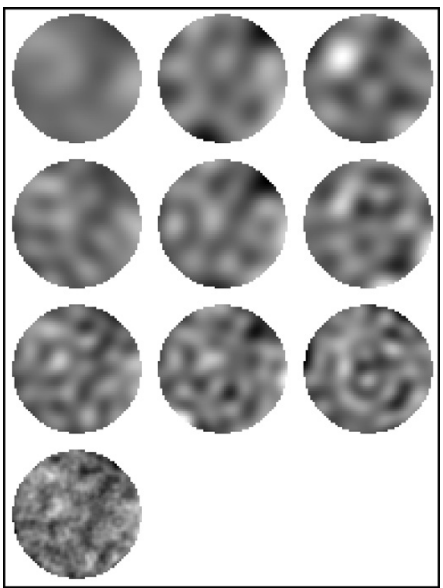

(c)

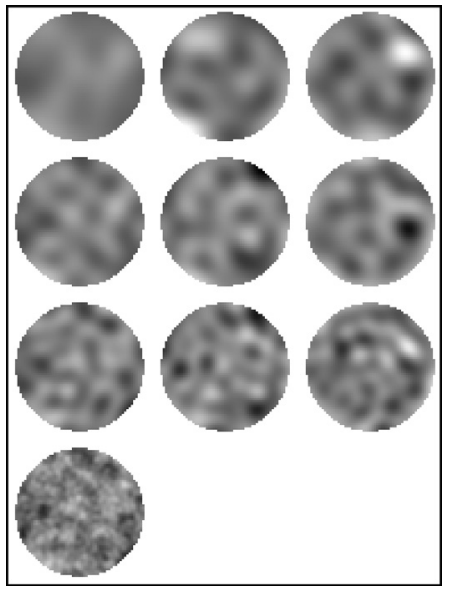

(d)

Fig. 9. Sample wavefronts from the simulation experiments. a)-d): Different realizations of the Kolmogorov statistics. Within each sub-figure, all wavefronts are displayed with identical scaling. Left: True wavefronts; Center: JPDS estimated wavefronts; Right: MFBD estimated wavefronts. 1st row: 35 KL modes; 2nd row: 50 modes; 3rd row: 100 modes; 4th row: All 1001 modes.

images, even with 10 frames, in bad seeing $\left(r_{0}=7 \mathrm{~cm}\right)$ and using only 35 aberration parameters. This re-enforces our earlier conclusion, that details of the high-order part of the wavefront are not important as long as it has the "right" statistical properties. In particular, the "speckles" seen in the wings of the PSF of Fig. 1 (upper row), have a small effect on the restored images. The excellent result obtained with $\hat{S}$ compensation based on "true" wavefronts also is consistent with the results discussed previously: that MFBD processing with 35 aberration parameters can produce transfer functions equivalent to those obtained with compensation by 100 aberration parameters, even though the actual wavefronts derived are demonstratedly wrong.

With MFBD processing, the improvement from $\hat{S}$ compensation varies strongly with the number of aberration parameters, $M$. The remarkably good results obtained without $\hat{S}$ compensation for $M=35$ and $M=50$ constitutes a problem with $\hat{S}$ compensation! This is because our calculations of $\hat{S}$ do not take into account the compensation for high-order aberrations already provided by the MFBD (and JPDS) processing. This leads to an overcompensation of the effects of high-order aberrations for $M=35$ and $M=50$. This compensation is much smaller with $M=100$. With JPDS processing (panels c, f, i and l), the results show improved consistency. Here, $\hat{S}$ compensation with $M=50$ or $M=100$ leads to rms intensity errors that are reduced by a factor $2-3$. The obtained rms errors are consistently smaller than for MFBD processing without $\hat{S}$ compensation, when noise is present.

We have here demonstrated that MFBD image reconstruction by itself allows compensation effects by fitting transfer functions rather than wavefronts. In this sense, MFBD or JPDS processing provides more optimum image reconstruction than with a Shack-Hartmann based wavefront sensor. The main problem in the present context is that MFBD and (to a smaller extent) JPDS processing already introduces part of the compensation intended to be performed with $\hat{S}$. This leads to overcompensation if no counter measures are taken. By using JPDS processing, the compensation effects are constrained and the risk of overcompensation with $\hat{S}$ is small.

The relatively poor estimates of the wavefronts obtained with MFBD and JPDS processing may appear to contradict earlier simulations with phase diversity methods
(Löfdahl \& Scharmer 1994; Paxman et al. 1996), but are a direct consequence of the assumed $\mathrm{AO}$ correction, reducing the rms amplitudes of the first $35 \mathrm{KL}$ aberrations with a factor of 4. This leaves residual wavefronts that have small or negligible amplitudes for the first 35 modes and a correspondingly large contribution from higher-order modes. This leads to significant cross-talk from high-order aberrations, degrading the low-order wavefront estimates but actually leading to better estimates of the transfer functions than expected on the basis of the number of aberration parameters included. Figure 9 shows the wavefronts corresponding to the first 4 frames used to reconstruct the image with 10 frames. In the left column are shown (top to bottom) the true wavefronts represented with 35, 50, 100 and $1001 \mathrm{KL}$ modes. It is evident from the lower-left wavefront in each panel that the true wavefront has been stripped of its low-frequency wavefront components. It is also evident that the JPDS (mid column) and MFBD (right column) wavefront estimates show large differences and that JPDS provides the best estimate of the true wavefront.

\subsection{Inversion tests with point sources}

We have also made inversion tests with point sources. While not directly relevant for restoration of solar images, these tests do shed further light on the MFBD and JPDS compensation effects discussed above. The most striking result is that both MFBD and JPDS processing of synthetic noise-free point source images lead to nearly perfectly reproduced low-order wavefronts. To understand why granulation images and images of point sources lead to different wavefront estimates, we express the equation used to estimate the transfer function as follows: inserting the expression for $\hat{F}$ in Eq. (3) into Eq. (2), and replacing $D_{k}$ with $F T_{k}$, we obtain an expression for the error metric $L$ that corresponds to the scalar quantity minimized in MFBD and JPDS processing:

$L=\left.\sum_{u, v}|F|^{2} \sum_{k=1}^{K}\left|T_{k}-\hat{T}_{k} \sum_{n=1}^{K} T_{n} \hat{T}_{n}^{*}\right| \sum_{n=1}^{K}\left|\hat{T}_{n}\right|^{2}\right|^{2}$

Ignoring the complicated expression involving the exact and estimated transfer functions $T_{k}$ and $\hat{T}_{k}$, we emphasize that this expression is multiplied by the power spectrum of the object, $|F|^{2}$. 
For a point source, this power spectrum is unity at all spatial frequencies but for solar fine structure, the power spectrum falls off at high spatial frequencies. Depending on the object re-imaged, smaller or larger weight will be given to the high spatial frequencies, obviously leading to different results as regards the derived wavefronts but actually quite small differences in the derived transfer functions.

\subsection{Comment on the use of small subfields}

The results discussed above were all obtained with $128 \times 128$ pixel subfields. Calculations with $256 \times 256$-pixel subfields gave results that are virtually identical to those discussed. MFBD calculations with $80 \times 80$-pixel subfields gave poorer, but still acceptable, results. However, JPDS restorations with $80 \times 80$-pixel subfields essentially failed. This is attributed to the relatively large diameter of the PSF corresponding to the defocused images, causing problems from lack of information about the object outside the subfield and wrap-around effects when using FFTs to perform convolutions with the defocused PSF. It is thus more difficult to use small subfields with JPDS than with MFBD with the present methods.

\section{Tests with real data}

\subsection{Observations}

To test the proposed $\hat{S}$ compensation method we used granulation images recorded through a $0.44 \mathrm{~nm} F W H M$ 2-cavity filter centered at $630.26 \mathrm{~nm}$ and a $0.34 \mathrm{~nm}$ filter centered at $538.20 \mathrm{~nm}$, both used as wide-band channels for the SST/CRISP imaging spectro-polarimeter (Scharmer et al. 2008). The images were exposed by means of a rotating chopper, set to give exposure times of $16 \mathrm{~ms}$ and a dark read-out time of nearly $12 \mathrm{~ms}$, corresponding to an overall frame rate of $36 \mathrm{~Hz}$. The $630 \mathrm{~nm}$ and $538 \mathrm{~nm}$ data discussed here were recorded between 10:45 and 10:50 UT on 26 June 2009, during a period with reasonably good but strongly variable seeing. The science target for these observations was a small pore in AR 1023, located at approximately S22, W20, corresponding to a heliocentric distance of about $\theta=30^{\circ}(\mu=\cos \theta=0.87)$. This pore was also used as lock point for the AO system. However, in the present paper we discuss only field-free granulation outside this active region. The noise level in the recorded images was estimated from power spectra at high spatial frequencies and found to be about $0.9 \%$.

CRISP was used to repeatedly scan each of the $630.26 \mathrm{~nm}$ and $538.20 \mathrm{~nm}$ lines three times, using different numbers of wavelength positions for the two lines. These scans required a total of $17 \mathrm{~s}$ for the $630 \mathrm{~nm}$ line and $12 \mathrm{~s}$ for the $538 \mathrm{~nm}$ line, setting the time between pre-filter changes. Simultaneously, approximately 600 wide-band images were collected at $630 \mathrm{~nm}$ and 433 images at $538 \mathrm{~nm}$ during each scan. We refer to these $17 \mathrm{~s}$ and $12 \mathrm{~s}$ sets of wide-band images as "full scan" data sets but will primarily discuss the results of processing $2.2 \mathrm{~s}$ sub-sets of this data to match the time-scale of seeing changes.

The images were corrected for gain and bias and MFBD processed with the MOMFBD code, see Sect. 5.3 below.

\subsection{Seeing measurements}

Simultaneous measurements of Fried's parameter $r_{0}$ were obtained with the SST wide-field wavefront sensor (WFWFS; Scharmer \& van Werkhoven 2010), developed as part of an effort to characterize day-time high-altitude seeing at La Palma.
The WFWFS is mounted on a beam that splits off light immediately before the tip-tilt and deformable mirrors, such that $r_{0}$ can be measured through the telescope and from a FOV that is adjacent to the science FOV, but without impact from the AO system. Between UT 10:00 and 10:48, processed WFWFS data averaged over $100 \mathrm{~s}$ gave estimates of $r_{0}$ in the range $11-12 \mathrm{~cm}$ at $500 \mathrm{~nm}$, corresponding to $15-16 \mathrm{~cm}$ at $630 \mathrm{~nm}$ and $12-13 \mathrm{~cm}$ at $538 \mathrm{~nm}$. However, the science images, recorded with the AO system in closed loop, showed strong variations in image quality on time scales on the order of a few seconds. The WFWFS variances measured are proportional to $r_{0}^{-5 / 3}$ (Eqs. (7)-(8); Scharmer \& van Werkhoven 2010), such that averaging wavefront sensor data in variable seeing gives the largest weights to relatively poor seeing (small values of $r_{0}$ ). However, MFBD image restoration gives the highest weights to good images (large values of $r_{0}$ ). We therefore compare data recorded over such short time intervals that $r_{0}$ can be considered constant. We re-processed the WFWFS data in blocks of $2.2 \mathrm{~s}$, or about 10 WFWFS CCD frames. Figure 10 shows in the upper panel the variation of $r_{0}$ with time from UT 10:45-10:50, obtained from overlapping 2.2-s blocks of WFWFS data. The red and blue curves in this panel indicate the wavelengths at which the science images were recorded, at $630 \mathrm{~nm}$ and $538 \mathrm{~nm}$ respectively. In the mid panel is shown the variation of the rms contrast measured (see Sect. 5.4.1) for the $630 \mathrm{~nm}$ and $538 \mathrm{~nm}$ images after flat-fielding but without MOMFBD image restoration. Comparing the two panels, there is a clear correlation between $r_{0}$ and the measured rms contrast; even small and rapid variations are reproduced in detail. The bottom panel shows the correlation between all measured values of $r_{0}$ and the rms contrast. In this figure, we have scaled the $r_{0}$ values measured with the WFWFS at $500 \mathrm{~nm}$ to $538 \mathrm{~nm}$ and $630 \mathrm{~nm}$ by assuming that $r_{0}$ is proportional to $\lambda^{6 / 5}$. We have also multiplied all rms contrasts at $630 \mathrm{~nm}$ by an ad hoc factor 1.22 , roughly compensating for the wavelength dependence of the granulation contrast. The plotted values cover a range of $r_{0}$ from $4 \mathrm{~cm}$ to nearly $30 \mathrm{~cm}$ and rms contrast from $2.2 \%$ to $7.8 \%$ and show an excellent correlation. The dotted curve corresponds to variation of the rms contrast with $r_{0}$ obtained from the simulations shown in Fig. 5, but divided by a factor 1.85 to fit the data. This large factor in part comes from the assumed perfect compensation of the first $35 \mathrm{KL}$ modes, corresponding to an $\mathrm{AO}$ system with $100 \%$ efficiency, and in part must be due to stray light.

Our conclusion from the excellent correlation between measured variations of $r_{0}$ with the WFWFS (bypassing the AO system) and the science images (recorded with the AO system in closed loop) is that the WFWFS indeed provides an accurate measure of seeing, although we cannot from this data rule out systematic errors, such as a scale factor error or a systematic bias, in the measured $r_{0}$ values.

\subsection{MFBD processing}

The MOMFBD software package was used to divide the images into $128 \times 128$-pixel overlapping subfields, which were separately MFBD restored using the $M=100$ most significant KL modes. The restored subfields were then mosaicked to produce restored images over the entire observed FOV. To investigate the effects of strongly variable seeing on the MFBD processing, we processed the data in blocks corresponding to about $2.2 \mathrm{~s}$ of data as well as in blocks corresponding to full scans (12 s resp. $17 \mathrm{~s}$ data). To allow a comparison between the two methods of processing, subsets of the full scan MFBD wavefronts and observed 

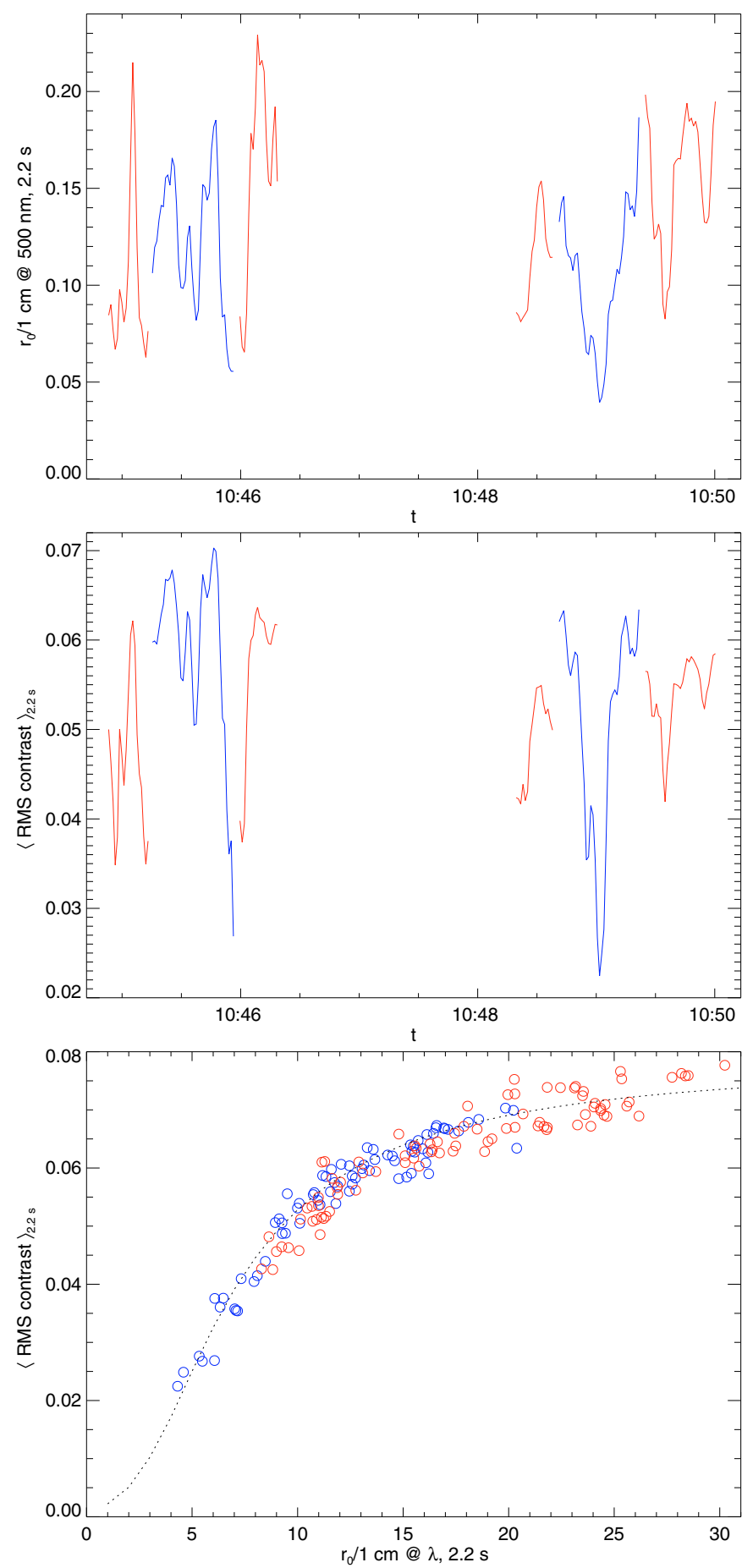

Fig. 10. Variation of Fried's parameter $r_{0}$ with time, measured with the SST wide-field wavefront sensor bypassing the AO system (top panel) and the corresponding variation of the measured granulation rms contrast of the science images (mid panel) obtained at $\lambda=538 \mathrm{~nm}$ (blue curves) and at $\lambda=630 \mathrm{~nm}$ (red curves) with the AO system in closed loop. The bottom panel shows the correlation between $r_{0}$ and the rms contrast at the two wavelengths but with the contrast at $\lambda=630 \mathrm{~nm}$ multiplied by 1.22 to bring the data onto a common curve. The dotted curve corresponds to the contrast of simulated data shown in Fig. 5, divided by a factor 1.85 .

images were used to restore the same raw images as used with the $2.2 \mathrm{~s}$ data.

The observed images are over-sampled by $12 \%$ at $630 \mathrm{~nm}$ but $4 \%$ under-sampled at $538 \mathrm{~nm}$. In the MOMFBD code as well

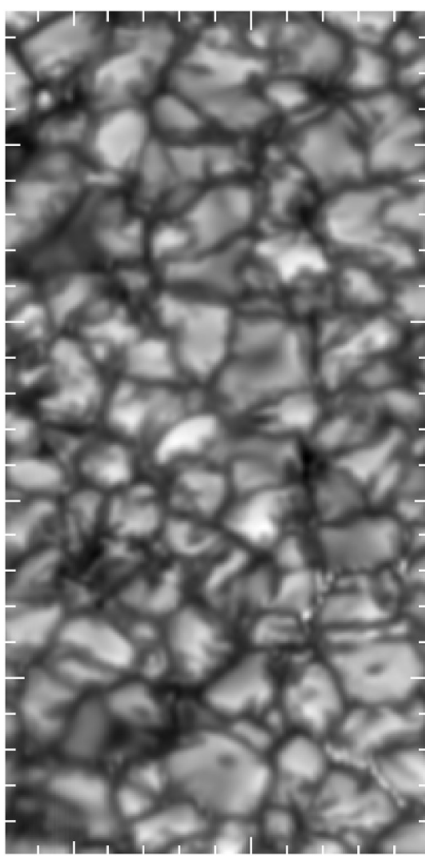

(a) $538 \mathrm{~nm}$

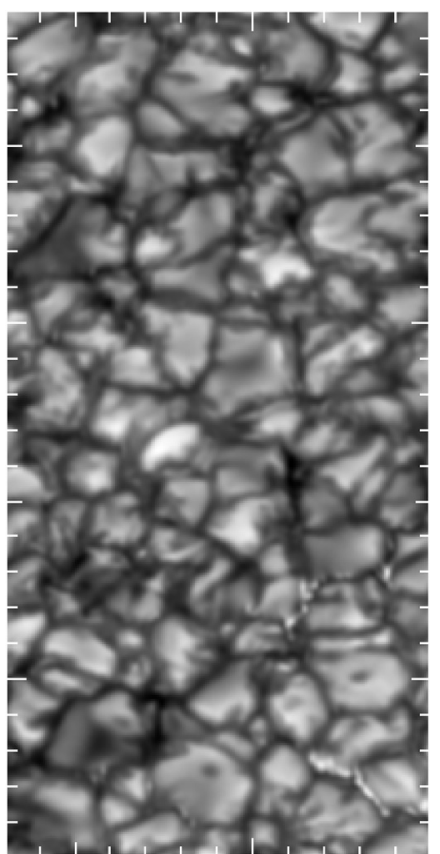

(b) $630 \mathrm{~nm}$
Fig. 11. Examples of MFBD restored images based on data collected at $\mu=0.87$ UT 10:50). The $200 \times 400$-pixel subfield shown was used for rms contrast measurements. Tick marks are $1^{\prime \prime}$ apart.

as in the $\hat{S}$ correction, under-sampling is implemented such that the phase over the entire pupil can be represented but the transfer function $\hat{T}_{k}$ is cropped at the Nyquist frequency.

\subsection{Results}

\subsubsection{Granulation contrast measurements}

Granulation contrast was measured over the $200 \times 400$-pixel subfield shown in Fig. 11. This region appears reasonably free from strong magnetic fields, as judged by the near absence of bright points and other sub-granular structure.

The measured contrasts (blue: at $538 \mathrm{~nm}$; red: at $630 \mathrm{~nm}$, multiplied by 1.22) are shown in Fig. 12. The upper panel shows the contrasts measured from blocks of images recorded during $2.2 \mathrm{~s}$ intervals. The contrasts plotted in the bottom panel correspond to MFBD sets based on full scan data. The plus symbols refer to contrasts obtained after conventional MFBD processing with $100 \mathrm{KL}$ aberrations, the squares to contrasts obtained after compensation also of high-order aberrations. We note that the results are quite similar for the two types of MFBD processing when $r_{0}$ is large, as expected. Data processed with $2.2 \mathrm{~s}$ sets show a slow and gradual decrease of the rms contrast with decreasing $r_{0}$ after high-order compensation (squares). Inspection of the restored images shows that this decrease in contrast is associated with a small but noticeable decrease of image quality. For values of $r_{0}$ smaller than $9-10 \mathrm{~cm}$, this degradation is obvious as reduced spatial resolution. A possible explanation for the reduced rms contrast in poor seeing is the finite integration time used: a seeing layer moving at $10 \mathrm{~m} \mathrm{~s}^{-1}$ is displaced by $16 \mathrm{~cm}$ peak-to-valley $(5 \mathrm{~cm} \mathrm{rms})$ during a $16 \mathrm{~ms}$ exposure and will cause increased smearing of the wavefront with decreasing $r_{0}$.

For the full scan data sets obtained in strongly varying seeing conditions, the trend is quite the opposite to that seen with the 
G. B. Scharmer et al.: High-order aberration compensation with MFBD and PD image restoration techniques
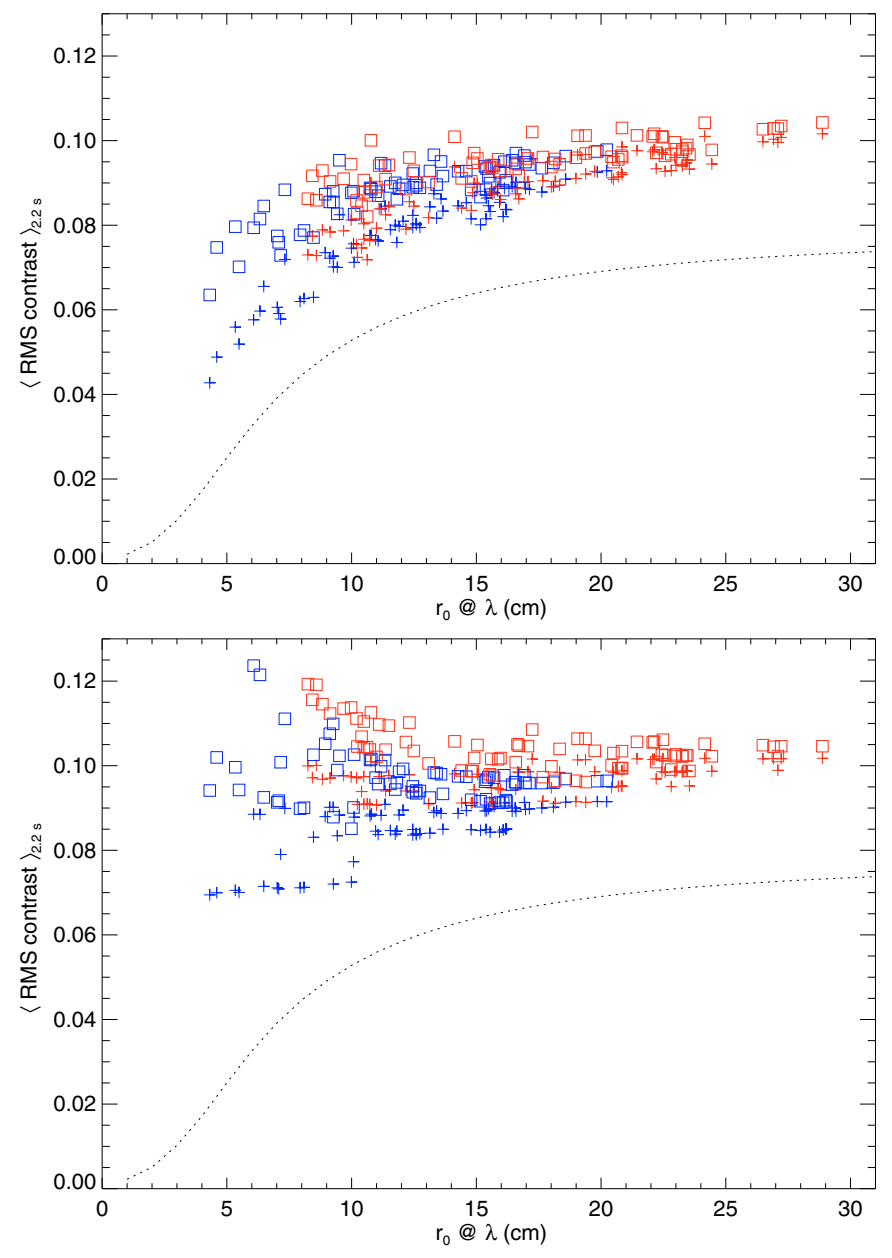

Fig. 12. The variation of granulation rms contrast with $r_{0}$ for MFBD restored images. Plus-symbols show the contrast after conventional MFBD processing, squares the result after $\hat{S}$-compensation. Red and blue symbols correspond to $630 \mathrm{~nm}$ and $538 \mathrm{~nm}$ resp., with the contrasts at $630 \mathrm{~nm}$ multiplied by a factor 1.22 . The upper panel shows the results from processing of images in blocks of $2.2 \mathrm{~s}$, the bottom panel from MFBD processing of the entire scan to determine the wavefronts, but with restoration of the images and high-order compensation carried out in $2.2 \mathrm{~s}$ blocks. The dotted curve is identical to that shown in the bottom panel of Fig. 10.

$2.2 \mathrm{~s}$ data sets. For these data, the rms contrast increases with decreasing $r_{0}$ after $\hat{S}$ compensation. This is again explained by the compensation effects discussed in Sect. 4.3.1: MFBD processing assumes that a unique object $F$ (see Eq. (3)) is "responsible" for all observed images of a data set, and that this object can be estimated with a fixed number of aberration parameters for each of the observed images. In stable seeing, this works well. However, when the seeing is strongly variable, this causes inconsistencies. During moments of bad seeing, the missing highorder aberrations lead to such poorly represented wavefronts that the observed images are inconsistent with the images recorded in good seeing. MFBD compensates this inconsistency by increasing the amplitudes of the low-order aberrations in bad seeing. When compensating for the high-order aberrations in the final reconstruction of the images, the overestimated wavefront rms leads to contrast values that are too high when $r_{0}$ is small. Comparison between the restored images from the $2.2 \mathrm{~s}$ and full scan MFBD shows no differences in image quality apart from the differences in contrast; quite clearly either way of MFBD

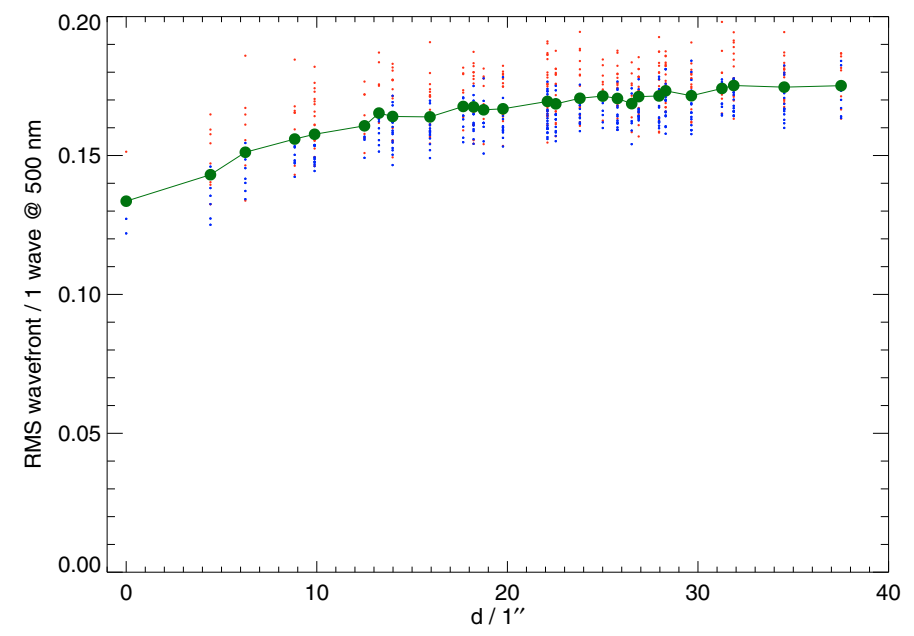

Fig. 13. The variation of the wavefront rms (in units of waves at $500 \mathrm{~nm}$ ) with distance $d$ from FOV center in arc seconds. MFBD restorations with $M=100$ corresponding to the bottom tile of Fig. 12. Blue: two sets of $538 \mathrm{~nm}$ data; Red: two sets of $630 \mathrm{~nm}$ data; Green: average of all four sets.

processing works very well as long as the number of images in the data set is not too small.

\subsubsection{Comparison with 3D MHD simulations}

Based on the results of the $2.2 \mathrm{~s}$ data sets, we are finally in a position to compare the measured granulation contrasts with those of simulations. The highest measured rms contrast is $9.8 \%$ at $538 \mathrm{~nm}$ and $8.5 \%$ at $630 \mathrm{~nm}$ (note that the contrast values at $630 \mathrm{~nm}$ in Fig. 12 are multiplied by 1.22).

To compare measured rms contrasts with those of the 3D simulations, we calculated a synthetic spectrum covering the Fe I $630.1 \mathrm{~nm}$ and $630.2 \mathrm{~nm}$ lines and nearby continuum, using the same simulation snapshot shown in Fig. 5 but for a heliocentric distance corresponding to $\mu=0.87$. Similarly, we included the weak $\mathrm{C}$ I line and two stronger Fe I lines for calculating a synthetic spectrum corresponding to the $538 \mathrm{~nm}$ observations. We multiplied the synthetic spectra obtained at each pixel with the transmission profiles of the CRISP pre-filters used to record the observed data and calculated the contrast of the granulation pattern. We obtained an rms contrast of $13.4 \%$, compared to $13.9 \%$ at a clean nearby continuum wavelength, for the $630 \mathrm{~nm}$ data and $17.3 \%$, compared to a continuum contrast of $17.8 \%$ for the $538 \mathrm{~nm}$ data. In comparison, the corresponding values at $\mu=1$ are $14.2 \%$ and $14.6 \%$ at $630 \mathrm{~nm}$ and $18.2 \%$ and $18.6 \%$ at $538 \mathrm{~nm}$. It should be emphasized that these rms contrasts have been calculated without degrading the spatial resolution of the synthetic data to that of a 1-m telescope, nor have any effects of noise on the data been included.

The measured rms contrasts are only $57 \%$ at $538 \mathrm{~nm}$ and $63 \%$ at $630 \mathrm{~nm}$ of those expected, clearly demonstrating the existence of sofar unidentified stray light sources and suggesting that this stray light increases at shorter wavelengths. The origin of this stray light will be investigated in forthcoming papers.

\subsubsection{Wavefronts}

Figure 13 shows the variation of the wavefront rms with distance from the center of the FOV, estimated from MFBD processing. The plot corresponds to two good data sets at each wavelength. Wavefront tip-tilt (KL coefficients 2 and 3) are not included in 

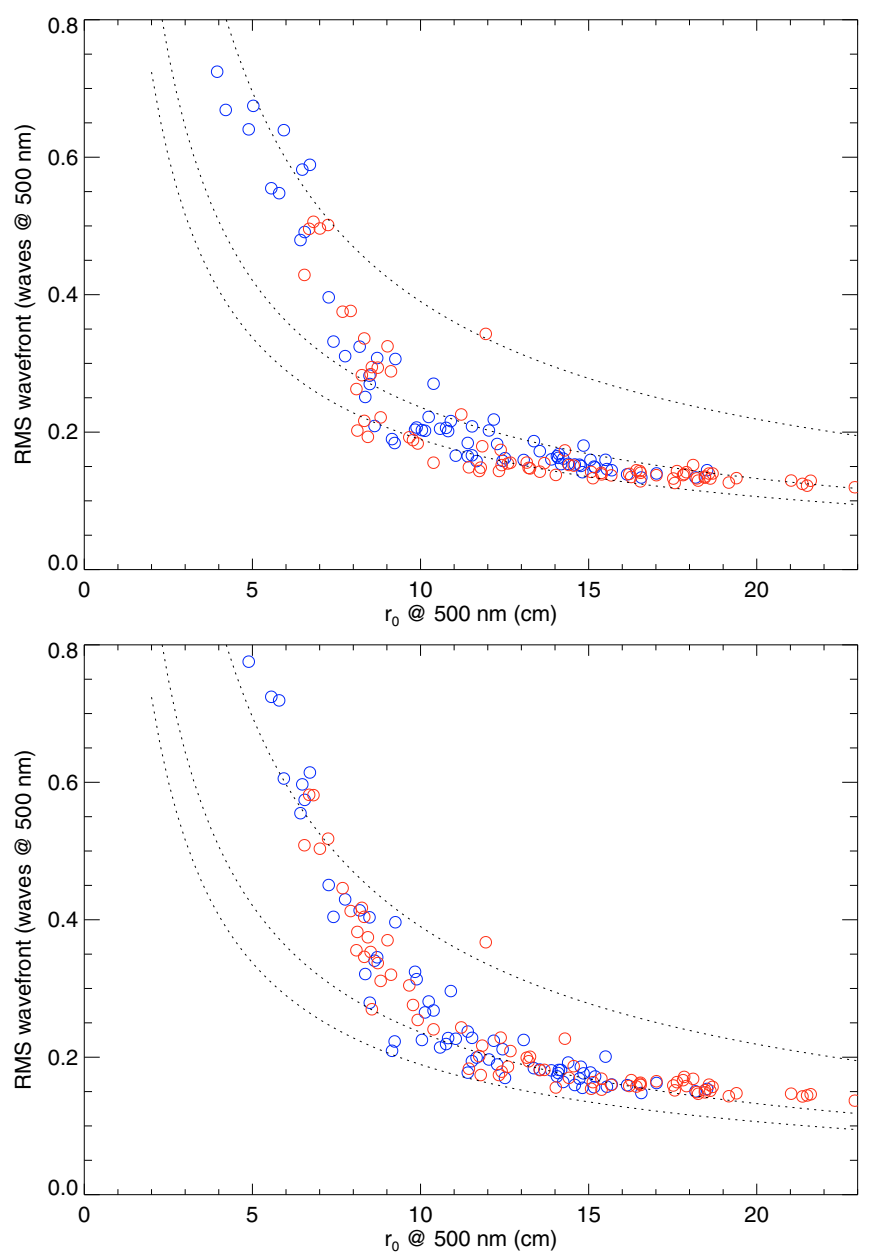

Fig. 14. RMS wavefronts vs. $r_{0}$. Red and blue circles: rms wavefronts as estimated with MFBD using $M=100$ modes and $2.2 \mathrm{~s}$ data blocks (top tile), and 12-17 s data blocks (bottom tile). Black dotted curves: Wavefront rms expected from perfect correction of the first $N-1$ modes for $N=3,10,17$ (top to bottom) KL modes.

the calculation. As expected, the wavefront rms is smallest at the center of the FOV $(d=0)$, where the AO is locked, and gradually increases away from the lock point. This demonstrates that in contrast to speckle techniques, MOMFBD techniques can be used with $\mathrm{AO}$ compensated images to retrieve wavefronts without any a priori knowledge of how anisoplanatism is introduced by the AO system and the Earth's atmosphere.

Figure 14 shows the variation of the MFBD estimates of the wavefront rms with $r_{0}$ at the center of the FOV, corresponding to the AO lock-point. The upper panel shows the wavefront rms obtained by MFBD processing of data in $2.2 \mathrm{~s}$ blocks, the bottom panel by processing full scan data. The three dotted curves correspond to the expected wavefront rms with the AO system perfectly compensating the first 2, 9 and $16 \mathrm{KL}$ aberrations (top to bottom, corresponding to $N=3,10$ and 17 in Eq. (6)). The top panel shows that the MFBD estimate of the wavefront rms is consistent with perfect $\mathrm{AO}$ compensation of about $15 \mathrm{KL}$ modes when $r_{0}$ is approximately 10-12 cm, whereas when $r_{0}$ is larger than $20 \mathrm{~cm}$, the rms wavefront approaches the values expected with perfect compensation of only $10 \mathrm{KL}$ modes. We conclude that the MFBD wavefront rms estimates are similar, although somewhat higher, than what we expect from the independent WFWFS estimates of $r_{0}$. This strongly suggests that the
WFWFS measurements of $r_{0}$ are at most associated with small systematic errors.

For values of $r_{0}$ smaller than about $9.5 \mathrm{~cm}$, the MFBD estimated wavefront rms shows a rapid transition to values equivalent to only 2 perfectly corrected KL modes. This corresponds to pure tip-tilt correction, or recording images with short exposures as used with the present data, but with the AO system not functioning at all. Such failure of solar AO systems to lock in poor seeing is well-known: When $r_{0}$ is smaller than the sub-aperture diameters of the Shack-Hartmann wavefront sensor controlling the AO system, the granulation images start to degrade, leading to image positions that are poorly determined with crosscorrelation techniques. The SST AO system uses fairly large sub-aperture diameters, $14 \mathrm{~cm}$, and is more vulnerable to bad seeing than other solar AO systems. The data in Fig. 14 suggest that the efficiency of the SST AO system starts to degrade when the ratio of $r_{0}$ to the sub-aperture diameter is about 0.7 . This ratio is similar to what was found for the WFWFS, using sub-aperture diameters of about $9.8 \mathrm{~cm}$, leading to strongly increased noise levels when $r_{0}$ is less than about $7 \mathrm{~cm}$ (Scharmer $\&$ van Werkhoven 2010).

The bottom panel in Fig. 14, based on full-scan data recorded in strongly variable seeing, shows a variation of wavefront rms with $r_{0}$ that is quite similar to that of the upper panel. However, the wavefront rms, in particular during bad seeing, is systematically higher for the full-scan data. This is consistent with the systematic differences in granulation contrasts found for these data, discussed in the previous section: In strongly varying seeing the limited number of aberrations used to model the wavefront becomes a problem in particular during moments of poor seeing. The MFBD compensates this by increasing the rms of the loworder aberrations. When combined with $\hat{S}$-compensation, this leads to overcompensation. At the same time, MFBD optimization is dominated by the best frames, because of the weighting of the transfer functions used to restore the object, see Eq. (4). This reduces the contribution of the poorer and overcompensated frames in the restored image and thus leads to a reduction of the overcompensation effects when images recorded in variable seeing are combined and restored.

\section{Stray-light compensation}

An in-depth discussion of stray light measurements and compensation is beyond the scope of the present paper. However, we note that the primary focal plane used to calibrate the control matrix of an AO system can also be used to aid stray light calibrations of any following optics and instrumentation. Such (partial) stray light calibrations can be made by locking the AO system on the stray light target and recording images with the science camera at several focus positions. Processing these images with JPDS techniques, allows the effects of any residual aberrations to be separated from those of stray light, making such measurements robust.

Spatial stray light can be simply modeled as a convolution:

$D_{k}=F T_{\mathrm{s}} T_{k}+N_{k}$,

where $T_{\mathrm{s}}$ is the Fourier transform of the stray light PSF, $t_{\mathrm{s}}$. The residual aberrations can be identified and determined by recording pinhole images at different focus locations and the corresponding transfer function $T_{k}$ can be calculated and compensated for with phase diversity techniques. The stray light transfer function $T_{\mathrm{s}}$ is assumed to be invariant with respect to the focus position. It is then evident that phase diversity image restoration 
will produce a restored "object" that is the product of $F$ and $T_{\mathrm{s}}$ and that $T_{\mathrm{s}}$ can therefore only be determined if the object $F$ is known. For example, using a circular pinhole as object, corresponds to a binary object that is of constant intensity within a radius $r$ and zero outside that radius. Recording images of pinholes of different diameters should allow the stray light PSF to be determined with confidence. Procedures for such stray light measurements and compensation will be described in a forthcoming paper ${ }^{4}$.

Of relevance to the present paper is that the stray light PSF, as well as $\hat{S}$ in bad seeing, have wings that can extend well beyond several arc sec. Proper compensation for such a broad PSF during MFBD/JPDS processing would cause problems with the small sub-fields needed to deal with anisoplanatism. The calculation of $\hat{S}$ involves atmospheric transfer functions modified by high-order aberrations but does not involve the observed images $d_{k}$. A reasonable expectation is that $\hat{S}$ should show relatively small and smooth variations over a large FOV. This would allow $\hat{S} T_{\mathrm{s}}$, averaged over a large fraction over the FOV, to compensate for the combined effects of high-order atmospheric aberrations and stray light using only a few deconvolutions, independent of the sub-fielding used in previous MFBD or JPDS processing. The restored images can then be properly compensated for stray light originating from the broad wings of the PSF corresponding to $\hat{S} T_{\mathrm{s}}$.

\section{Conclusions}

The development of techniques for restoration of solar images based on multi-frame blind deconcolution (MFBD) such as phase diversity (PD; Löfdahl \& Scharmer 1994), joint phase diversity speckle (JPDS; Paxman et al. 1992) and multi-object multi-frame blind deconvolution (MOMFBD; Löfdahl 2002; van Noort et al. 2005) is of major importance to present and future broad-band imaging, imaging spectrometry and imaging spectropolarimetry with ground-based solar telescopes. An important advantage of these techniques is that they do not rely on statistical properties of atmospheric seeing, including anisoplanatism introduced by the Earth's atmosphere and a conventional or multi-conjugate AO system. These techniques, implemented in the MOMFBD $\mathrm{C}++$ code developed by van Noort et al. (2005), have been used extensively to process data sets recorded with the SST. This has resulted in stunning time sequences with near-diffraction limited resolution. The remarkably stable image quality achieved when many exposed frames are processed as a single data set strongly indicates that this code is working well. The present work represents the first systematic evaluation of its performance.

Our simulations confirm and explain the excellent performance of the code with images recorded using a low-order (37 electrode) AO system. In particular, we demonstrate that even though MFBD processing of AO compensated images leads to poorly determined wavefronts, this is of secondary importance. The transfer functions, needed to restore images, are

\footnotetext{
${ }_{4}$ We remark that such a calibration will also include the detector MTF. This MTF is primarily the combination of two effects: The first is from the integration over the finite size of the pixels and the second is from charge diffusion in the silicon substrate of the CCD detector (e.g., Stevens \& Lavine 1994). With back-illuminated thinned CCDs operating at near infrared wavelengths, as used with CRISP, there is a third stray light source from light that is first transmitted through the thin silicon layer of the CCD and then scattered back into the beam. Compensation for such stray light needs special calibration and compensation techniques (de la Cruz Rodríguez et al., in preparation).
}

actually better determined than expected from the number of $\mathrm{KL}$ modes used to represent the wavefront. A surprising result is that the addition of a phase diversity (defocused) channel not necessarily improves the result when many images are processed simultaneously: adding such a channel constrains the wavefronts to more closely represent reality, but the obtained transfer functions may actually be more accurate without the defocused channel. From this perspective, MFBD processing rather than JPDS processing is preferrable. However, when applying $\hat{S}$-compensation to the restored images, the more accurate wavefronts of JPDS constitute an advantage. A disadvantage of JPDS processing with an added diversity channel is that defocused images are fuzzier, causing "leakage" of (stray-)light across subfield boundaries and wrapping around when using FFTs to perform convolutions. This degrades the quality of the restorations or even cause the inversions to fail when the subfields are small. We can thus not simply conclude that the MFBD or JPDS method of processing in general is preferrable, but always recording also defocused images is a good strategy since this allows either mode of processing the data.

A drawback of MFBD and JPDS techniques is that truncation of the wavefront expansion is necessary. This leaves a tail of uncompensated high-order aberrations and undercompensation of the restored images. Based on restorations of images recorded in strongly variable seeing, we have found that MFBD processing compensates for the missing high-order aberrations by increasing the amplitudes of the low-order aberrations. This compensation effect is particularly large during moments of bad seeing but the corresponding exposed frames are given relatively small weight in the restored images. This explains the apparent stable image quality of movies made from MFBD processed data.

We have proposed a method for further compensation of missing high-order aberrations, based on statistics of Kolmogorov turbulence. Our simulations show that such compensation in principle should allow perfect image restorations in good to excellent seeing conditions. A major advantage of the method is that very good results can be obtained even when only a small number of frames are used to restore an object. However, the high-order mode compensations already introduced by MFBD or JPDS image processing limits the overall improvement factor to about 2-3, which nevertheless should represent an important improvement of the photometric quality of the restored images. A problem is MFBD processing of data sets recorded during strongly variable seeing. In such conditions, the use of a truncated wavefront leads to overcompensation for the images recorded during moments of poor seeing such that the contrast of the restored images increases with decreasing $r_{0}$ when applying $\hat{S}$-compensation. By breaking up the processing in smaller data sets, these overcompensation effects are strongly reduced.

Using images recorded with the SST in $1^{\prime \prime}$ seeing, we have calculated the rms contrast of solar granulation. The obtained "raw" contrast values range from $7.5 \%$ at $538 \mathrm{~nm}$ and $6.5 \%$ at $630 \mathrm{~nm}$ for the best images, observed away from sun center (at $\cos \theta=\mu=0.87$ ) and through pre-filters that contain a few moderately strong Fe I lines. Due to the previous compensation by the AO system, the measured rms contrast increases by only a fairly small amount to $9 \%$ and $7.5 \%$ respectively for MFBD processed images using $100 \mathrm{KL}$ aberrations. Simultaneous measurements of Fried's parameter $r_{0}$ were used to aid compensation for high-order aberrations and yields an increase of the rms contrast to close to $10 \%$ at $538 \mathrm{~nm}$ and $8.5 \%$ at $630 \mathrm{~nm}$. The corresponding rms contrast of synthetic images obtained from $3 \mathrm{D}$ 
MHD simulations, taking into account the strongest lines within the filters used and an inclination angle of $\theta=30^{\circ}$, is $17.4 \%$ at $538 \mathrm{~nm}$ and $13.3 \%$ at $630 \mathrm{~nm}$. This implies a remaining discrepancy of $37-43 \%$ of the true contrast, the major part of which must be from stray light in the Earth's atmosphere, the telescope, the subsequent re-imaging optics, the finite $(16 \mathrm{~ms})$ integration time used to record the images and/or anisoplanatism, scintillation and image scale variations from high-altitude seeing. The origin of this missing stray light will be the topic of forth-coming papers.

Acknowledgements. Part of this work was supported by a Marie Curie Early Stage Research Training Fellowship of the European Community's Sixth Framework Programme under contract number MEST-CT-2005-020395: The USO-SP International School for Solar Physics. This work was also partially supported by the European Commission through the collaborative project 212482 "EST: the large aperture European Solar Telescope" Design Study (FP7 - Research Infrastructures) and by a planning grant from the Swedish Research Council.

M.G.L. is grateful to George (Guang-ming) Dai for sharing the his calculated radial Karhunen-Lòeve functions, used for the atmospheric turbulence simulations. The Swedish 1-m Solar Telescope is operated on the island of La Palma by the Institute for Solar Physics of the Royal Swedish Academy of Sciences in the Spanish Observatorio del Roque de los Muchachos of the Instituto de Astrofísica de Canarias.

\section{References}

Conan, J. M., Madec, P. Y., \& Rousset, G. 1992, in ESO Conference and Workshop Proceedings, Progress in Telescope and Instrumentation Technologies, ed. M.-H. Ulrich, 42, 475
Dai, G.-M. 1995, J. Opt. Soc. Am. A, 12, 2182

Danilovic, S., Gandorfer, A., Lagg, A., et al. 2008, A\&A, 484, L17

Fried, D. L. 1978, J. Opt. Soc. Am., 68, 1651

Lagg, A., Woch, J., Krupp, N., \& Solanki, S. K. 2004, A\&A, 414, 1109

Löfdahl, M. G. 2002, in Image Reconstruction from Incomplete Data II, ed. P. J.

Bones, M. A. Fiddy, \& R. P. Millane, Proc. SPIE, 4792, 146

Löfdahl, M. G., \& Scharmer, G. B. 1994, A\&AS, 107, 243

Marino, J., Rimmele, T. R., \& Christou, J. C. 2004, in Advancements in Adaptive Optics, ed. D. Bonaccini Calia, B. L. Ellerbroek, \& R. Ragazzoni, Proc. SPIE, 5490, 184

Mathew, S. K., Zakharov, V., \& Solanki, S. K. 2009, A\&A, 501, L19

Nordlund, Å., Stein, R. F., \& Asplund, M. 2009, Liv. Rev. Sol. Phys., 6, 2

Paxman, R. G., Schulz, T. J., \& Fienup, J. R. 1992, J. Opt. Soc. Am. A, 9, 1072

Paxman, R. G., Seldin, J. H., Löfdahl, M. G., Scharmer, G. B., \& Keller, C. U. 1996, ApJ, 466, 1087

Puschmann, K. G., \& Sailer, M. 2006, A\&A, 454, 1011

Roddier, F. 1999, in Adaptive Optics in Astronomy, ed. F. Roddier (Cambridge, UK: Cambridge university press), 25

Rouppe van der Voort, L. H. M., Löfdahl, M. G., Kiselman, D., \& Scharmer, G. B. 2004, A\&A, 414, 717

Scharmer, G. B., \& van Werkhoven, T. I. M. 2010, A\&A, 513, A25

Scharmer, G. B., Narayan, G., Hillberg, T., et al. 2008, ApJ, 689, L69

Stein, R. F., \& Nordlund, A. 1998, ApJ, 499, 914

Stevens, E. G., \& Lavine, J. P. 1994, IEEE transactions on electronic devices, 41, 1753

Uitenbroek, H., Tritschler, A., \& Rimmele, T. 2007, ApJ, 668, 586

van Noort, M., Rouppe van der Voort, L., \& Löfdahl, M. G. 2005, Sol. Phys., 228,191

Veran, J., Rigaut, F., Maitre, H., \& Rouan, D. 1997, J. Opt. Soc. Am. A, 14, 3057 Wedemeyer-Böhm, S. 2008, A\&A, 487, 399

Wedemeyer-Böhm, S., \& Rouppe van der Voort, L. 2009, A\&A, 503, 225

Wöger, F. 2007, PhD Thesis, Kiepenheuer-Institut für Sonnenphysik AlbertLudwigs-University, Freiburg, Germany 